\title{
Variational Properties of a Generic Model Equation in Exterior 3D Domains
}

\author{
By
}

S. Kračmar and P. Penel

\begin{abstract}
We study a generic model equation in an exterior domain. We assume the case of a non-constant coefficient function. Using a variational approach we prove existence and uniqueness theorems in anisotropically weighted Sobolev spaces. As the main tool we derive and apply an inequality of the Friedrichs-Poincare type.

Key Words and Phrases. Existence, Uniqueness, Variational Method, Anisotropically weighted Sobolev spaces.

2000 Mathematics Subject Classification Numbers. Primary 35J20, Secondary 35D05, 35B45.
\end{abstract}

\section{Introduction}

\subsection{A scalar model problem}

In a three-dimensional exterior domain $\Omega$ in $\boldsymbol{R}^{3}$, the classical Oseen problem [15] describes the velocity vector and the associated pressure by a linearized version of the incompressible Navier-Stokes equations as a perturbation of $v_{\infty}$ the velocity at infinity; $v_{\infty}$ is generally assumed to be constant in a fixed direction, say the first axis, $v_{\infty}=\left|v_{\infty}\right| e_{1}$. In the next we denote $\left|v_{\infty}\right|$ by $k$, and we will write the convective term in the form $k \partial_{1} v$. On the other hand it is known that for various flows past a rotating obstacle, the convective term is modified by some additional term $a \cdot \nabla v$, where $a=a(x)$ is some concrete nonconstant coefficient function, e.g. $\omega \times x$ with a given vector $\omega$, see $[6,12]$; in view of industrial applications $a(x)$ can also play the role of an experimentally known standard, see [7].

This paper is devoted to the study of the following boundary problem:

$$
\begin{gathered}
-v \Delta u+k \partial_{1} u+a \cdot \nabla u=f \quad \text { in } \Omega, \\
u=0 \quad \text { on } \partial \Omega, \\
u \rightarrow 0 \quad \text { as }|x| \rightarrow \infty,
\end{gathered}
$$

where $v$ and $k$ are some positive constants, $a=a(x)$ a given vector function, $f=f(x)$ a given scalar function.

The elliptic equation (1.1) can be taken as a scalar model equation for the Oseen equations in the steady case: 


$$
\begin{aligned}
-v \Delta v+k \partial_{1} v+a \cdot \nabla v+\nabla p & =f^{\prime} \quad \text { in } \Omega, \\
\nabla \cdot v & =0 \quad \text { in } \Omega .
\end{aligned}
$$

In the exterior domain $\Omega$, the convective operators $k \partial_{1}$ and $a \cdot \nabla$ cannot be treated as perturbations of lower order of the Laplacian, this is well known.

A common approach to study the asymptotic properties of the solutions to the Dirichlet problem of the classical steady Oseen flow (the case $a(x) \equiv 0$ ) is to use convolutions with Oseen fundamental tensor and its first and second gradients for the velocity (or with the fundamental solution of Laplace equation for the pressure), see e.g. $[3,10,11,8]$. So, the situation is completely different in the study of our model equation. But it is also crucial to understand the anisotropic structure of the solutions near the infinity in this case using the following weight functions

$$
\begin{gathered}
w(x)=\eta_{\beta}^{\alpha}(x)=\eta_{\beta}^{\alpha}(x ; \delta, \varepsilon)=(1+\delta r)^{\alpha}(1+\varepsilon s)^{\beta}, \\
r \equiv r(x)=\left(x_{1}^{2}+x_{2}^{2}+x_{3}^{2}\right)^{1 / 2}, \quad s \equiv s(x)=r-x_{1},
\end{gathered}
$$

for $x=\left[x_{1}, x_{2}, x_{3}\right] \in \boldsymbol{R}^{3}, \varepsilon, \delta>0, \alpha, \beta \in \boldsymbol{R}$. Discussing the range of the exponents $\alpha$ and $\beta$ the corresponding weighted spaces $L^{p}(\Omega ; w)$ give the appropriate framework to test the solutions to $(1.1)-(1.3)$ also with $a(x) \not \equiv 0$. This paper is concerned with $p=2$.

Let us mention that $\eta_{\beta}^{\alpha}$ belongs to the Muckenhoupt class $A_{2}$ of weights in $\boldsymbol{R}^{3}$ if $-1<\beta<1$ and $-3<\alpha+\beta<3$.

\subsection{Basic notations and elementary properties}

Let us outline our notations: The domain $\Omega$ is exterior to a body $\Omega_{c}$, i.e. $\Omega=\boldsymbol{R}^{3} \backslash \Omega_{c}$, we assume $\Omega_{c}$ to be compact with a Lipschitz boundary $\partial \Omega_{c}$. Let $0 \in \Omega_{c}$ and denote by $m>0$ the distance of $\partial \Omega_{c}$ to the origin $\operatorname{dist}\left(0, \partial \Omega_{c}\right)$, and by $M>0$ a real number such that $B^{M} \subset \Omega$, where $B^{R}=\left\{x \in \boldsymbol{R}^{3}\right.$, $|x| \geq R\}$. We need to denote the special sets $\Omega^{R}=\Omega \cap B^{R}, \Omega_{R}=\Omega \cap B_{R}$, where $B_{R}=\left\{x \in \boldsymbol{R}^{3} ;|x| \leq R\right\}, \quad B_{R}^{R_{0}}=B^{R_{0}} \cap B_{R}, \quad \Omega_{R}^{R_{0}}=\Omega_{R} \cap \Omega^{R_{0}}$ for positive numbers $R_{0}<R$.

Let $L^{2}(\Omega ; w)$ be the set of measurable functions $f(\cdot)$ on $\Omega$ such that

$$
\|f\|_{2, \Omega ; w}=\left(\int_{\Omega}|f|^{2} w d x\right)^{1 / 2}<\infty .
$$

We will use the notation $L_{\alpha, \beta}^{2}(\Omega)$ instead of $L^{2}\left(\Omega ; \eta_{\beta}^{\alpha}\right)$ and $\|\cdot\|_{2, \alpha, \beta}$ instead of $\|\cdot\|_{L^{2}\left(\Omega ; \eta_{\beta}^{\alpha}\right)}$. Because $\left(\eta_{\beta}^{\alpha}\right)^{-1}$ is locally integrable, then, by Hölder's inequality, it follows that $L_{\alpha, \beta}^{2}(\Omega) \subset L_{l o c}^{1}(\Omega)$. It thus makes sense to talk about weak derivatives of functions in $L_{\alpha, \beta}^{2}(\Omega)$. Let us define the weighted Sobolev 
space $H^{1}\left(\Omega ; \eta_{\beta_{0}}^{\alpha_{0}}, \eta_{\beta_{1}}^{\alpha_{1}}\right)$ as the set of functions $u \in L_{\alpha_{0}, \beta_{0}}^{2}(\Omega)$ with the weak derivatives $\partial_{i} u \in L_{\alpha_{1}, \beta_{1}}^{2}(\Omega)$. The norm of $u \in H^{1}\left(\Omega ; \eta_{\beta_{0}}^{\alpha_{0}}, \eta_{\beta_{1}}^{\alpha_{1}}\right)$ is given by

$$
\|u\|_{H^{1}\left(\Omega ; \eta_{\beta_{0}}^{\left.\alpha_{0}, \eta_{\beta_{1}}^{\alpha_{1}}\right)}\right.}=\left(\int_{\Omega}|u|^{2} \eta_{\beta_{0}}^{\alpha_{0}} d x+\int_{\Omega}|\nabla u|^{2} \eta_{\beta_{1}}^{\alpha_{1}} d x\right)^{1 / 2} .
$$

As usual, $\stackrel{\circ}{H}^{1}\left(\Omega ; \eta_{\beta_{0}}^{\alpha_{0}}, \eta_{\beta_{1}}^{\alpha_{1}}\right)$ will be the closure of $C_{0}^{\infty}(\Omega)$ in $H^{1}\left(\Omega ; \eta_{\beta_{0}}^{\alpha_{0}}, \eta_{\beta_{1}}^{\alpha_{1}}\right)$. The following proposition is proved exactly in the same way as in the non-weighted case (see R. A. Adams [1, pp. 45-46]), when $\eta_{\beta}^{\alpha} \in A_{2}$.

Proposition 1.1. Let $\Omega \subset \boldsymbol{R}^{3}$ be open set and $\eta_{\beta_{0}}^{\alpha_{0}}, \eta_{\beta_{1}}^{\alpha_{1}} \in A_{2}$. Then the Sobolev spaces $H^{1}\left(\Omega ; \eta_{\beta_{0}}^{\alpha_{0}}, \eta_{\beta_{1}}^{\alpha_{1}}\right)$ and $\stackrel{\circ}{H}^{1}\left(\Omega ; \eta_{\beta_{0}}^{\alpha_{0}}, \eta_{\beta_{1}}^{\alpha_{1}}\right)$ are Banach spaces, more precisely Hilbert spaces equipped with the scalar product

$$
(u, v)_{H^{1}\left(\Omega ; \eta_{\beta_{0}}^{\alpha_{0}}, \eta_{\beta_{1}}^{\alpha_{1}}\right)}=\int_{\Omega} u v \eta_{\beta_{0}}^{\alpha_{0}} d x+\int_{\Omega} \nabla u \cdot \nabla v \eta_{\beta_{1}}^{\alpha_{1}} d x
$$

For simplicity, we shall use the following abbreviations:

$$
\begin{array}{lll}
H^{1}(\Omega) & \text { instead of } & H^{1}(\Omega ; 1,1) \\
\stackrel{\circ}{H}^{1}(\Omega) & \text { instead of } & \stackrel{\circ}{H}^{1}(\Omega ; 1,1) \\
L_{\alpha, \beta}^{2}(\Omega) & \text { instead of } & L^{2}\left(\Omega ; \eta_{\beta}^{\alpha}\right) \\
\|\cdot\|_{2, \alpha, \beta} & \text { instead of } & \|\cdot\|_{L^{2}\left(\Omega ; \eta_{\beta}^{\alpha}\right)} \\
\stackrel{\circ}{H_{\alpha, \beta}^{1}}(\Omega) & \text { instead of } & \stackrel{\circ}{H^{1}}\left(\Omega ; \eta_{\beta-1}^{\alpha-1}, \eta_{\beta}^{\alpha}\right) \\
V_{\alpha, \beta}(\Omega) & \text { instead of } & \stackrel{\circ}{H^{1}}\left(\Omega ; \eta_{\beta}^{\alpha-1}, \eta_{\beta}^{\alpha}\right)
\end{array}
$$

In fact we shall only use these last two Hilbert spaces for $\alpha \geq 0, \beta>0$, $\alpha+\beta<3$. Concerning the weight functions $\eta_{\beta}^{\alpha}$, we will use two notations $\eta_{\beta}^{\alpha}(x)$ and $\eta_{\beta, \varepsilon}^{\alpha, \delta}(x)$ taking the advantages of the following formulas and remarks:

Remark 1.2. Let us note that for $\eta_{\beta, \varepsilon}^{\alpha, \delta}$ and for any $\delta_{1}, \delta_{2}, \varepsilon_{1}, \varepsilon_{2}>0$ one has

$$
\begin{aligned}
& c_{\min } \cdot \eta_{\beta, \varepsilon_{2}}^{\alpha, \delta_{2}} \leq \eta_{\beta, \varepsilon_{1}}^{\alpha, \delta_{1}} \leq c_{\max } \cdot \eta_{\beta, \varepsilon_{2}}^{\alpha, \delta_{2}}, \\
& \text { where } \quad c_{\min }=\min \left(1,\left(\delta_{1} / \delta_{2}\right)^{\alpha}\right) \cdot \min \left(1,\left(\varepsilon_{1} / \varepsilon_{2}\right)^{\beta}\right), \\
& c_{\max }=\max \left(1,\left(\delta_{1} / \delta_{2}\right)^{\alpha}\right) \cdot \max \left(1,\left(\varepsilon_{1} / \varepsilon_{2}\right)^{\beta}\right) .
\end{aligned}
$$

The parameters $\delta$ and $\varepsilon$ are useful to re-scale separately the isotropic and anisotropic parts of weight function $\eta_{\beta}^{\alpha}$. We also have the following elementary inequalities for $\beta>0$ :

$$
2^{\min (0, \beta-1)}\left[1+(\varepsilon \cdot s)^{\beta}\right] \leq \eta_{\beta, \varepsilon}^{0, \delta} \leq 2^{\max (0, \beta-1)}\left[1+(\varepsilon \cdot s)^{\beta}\right]
$$


Remark 1.3. Obviously we have the explicit expressions:

$$
\partial_{i} r \equiv \frac{\partial r}{\partial x_{i}}=\frac{x_{i}}{r}, \quad \partial_{1} s=\frac{-s}{r},
$$

$$
\nabla r \cdot \nabla r \equiv 1, \quad \nabla s \cdot \nabla r=\frac{s}{r}, \quad \nabla s \cdot \nabla s=\frac{2 s}{r}, \quad \Delta r=\Delta s=\frac{2}{r},
$$

$$
\begin{aligned}
& \partial_{1} \eta_{\beta}^{\alpha}=\left\{\alpha \delta(1+\varepsilon s) \frac{x_{1}}{r}-\beta \varepsilon(1+\delta r) \frac{s}{r}\right\} \eta_{\beta-1}^{\alpha-1}, \\
& \nabla \eta_{\beta}^{\alpha}=\{\alpha \delta(1+\varepsilon s) \nabla r+\beta \varepsilon(1+\delta r) \nabla s\} \eta_{\beta-1}^{\alpha-1}, \\
& \left|\nabla \eta_{\beta}^{\alpha}\right|^{2}=\left\{\alpha^{2} \delta^{2}\left(\frac{1+\varepsilon s}{1+\delta r}\right)+2 \alpha \beta \delta \varepsilon \frac{s}{r}\right. \\
& \left.+2 \beta^{2} \varepsilon^{2}\left(\frac{1+\delta r}{1+\varepsilon s}\right) \frac{s}{r}\right\}\left(\eta_{\beta-1 / 2}^{\alpha-1 / 2}\right)^{2} \\
& \Delta \eta_{\beta}^{\alpha}=\left\{\alpha(\alpha-1) \delta^{2}\left(\frac{1+\varepsilon s}{1+\delta r}\right)+2 \alpha \beta \delta \varepsilon \frac{s}{r}\right. \\
& +2 \beta(\beta-1) \varepsilon^{2}\left(\frac{1+\delta r}{1+\varepsilon s}\right) \frac{s}{r} \\
& \left.+2 \alpha \delta(1+\varepsilon s) \frac{1}{r}+2 \beta \varepsilon(1+\delta r) \frac{1}{r}\right\} \eta_{\beta-1}^{\alpha-1} .
\end{aligned}
$$

\subsection{Main results}

The weighted estimates of the solution to the stationary Oseen problem were firstly obtained by Finn, see [4], and then improved by Farwig [2]. In the case $a(x) \equiv 0$, Farwig studied the model equation (1.1), the way he proposed in [2] combines a variational approach with the application of the potential theory.

For the case of non-constant function $a(\cdot)$ we cannot follow the same way because we have no expression of the fundamental solution. We are also motivated by a Farwig's remark ([2], Remark 2.8 p. 457) to improve his variational result for positive $\alpha$.

In solving the problem (1.1)-(1.3) by means of a pure variational approach, we shall deal with the following equation:

$$
\begin{gathered}
v \int_{\Omega}|\nabla u|^{2} w d x+v \int_{\Omega} u \nabla u \cdot \nabla w d x-\frac{k}{2} \int_{\Omega} u^{2} \partial_{1} w d x \\
-\frac{1}{2} \int_{\Omega} u^{2} \operatorname{div}(w a) d x=\int_{\Omega} f u w d x
\end{gathered}
$$


as we get integrating formally the product of (1.1) with $w u$ ( $w$ is an appropriate weight function). The left-hand side can be estimated from below by

$$
\frac{v}{2} \int_{\Omega}|\nabla u|^{2} w d x-\frac{1}{2} \int_{\Omega} u^{2}\left(v \frac{|\nabla w|^{2}}{w}+k \partial_{1} w+\operatorname{div}(w a)\right) d x .
$$

It is not difficult to observe the existence of $s_{0}>0$ such that $-v\left(|\nabla w|^{2} / w\right)-$ $k \partial_{1} w \geq 0$ for $w=\eta_{\beta}^{\alpha}$ and $s \geq s_{0}$, (see Appendix A). Moreover, because this term is known explicitly, we have the possibility to evaluate it from below by a small negative quantity in the form $-C \eta_{\beta-1}^{\alpha-1}$ without any constraint in $s(\cdot)$ (see Lemma 3.1).

An improved weighted Friedrichs-Poincaré type inequality in $\stackrel{\circ}{H}_{\alpha, \beta}^{1}(\Omega)$ is necessary: it is the first main technical result of this paper. The obtained inequality allows us to compensate by the viscous Dirichlet integral the "small" negative contribution in the second integral of (1.10). We propose a similar treatment to control the "small" negative contribution caused by $a \cdot \nabla u$ in the third integral of (1.10), under appropriate conditions on $\operatorname{div}(w a)$ (see Lemma 3.4). We finally prove the existence of a weak solution (1.1)-(1.3) in $V_{\alpha, \beta}(\Omega)$ by the Lax-Milgram theorem.

The main results can be summarized in the following theorems $(\Omega$ is an exterior domain in $\boldsymbol{R}^{3}$ and parameters $\alpha, \beta, \delta, \varepsilon$ are specified in Section 1.2):

Theorem 1.4. Let $\beta>0$. There are positive constants $R_{0}, c_{0}, c_{1}$ depending on $\alpha, \beta, \delta, \varepsilon$ (explicit expressions of these constants are given by Lemma 2.3, essentially $c_{0}=O\left(\varepsilon^{-2}+\delta^{-2}\right)$ and $c_{1}=O\left(\varepsilon^{-1} \delta^{-1}\right)$ for $\delta$ and $\varepsilon$ tending to zero) such that

$$
\|v\|_{2, \alpha-1, \beta-1}^{2} \leq c_{0} \int_{\Omega_{R_{0}}}|\nabla v|^{2} \eta_{\beta}^{\alpha} d x+c_{1} \int_{\Omega^{R_{0}}}|\nabla v|^{2} \eta_{\beta}^{\alpha} d x
$$

for all $v \in \stackrel{\circ}{H}_{\alpha, \beta}^{1}(\Omega)$.

Theorem 1.5. Let $\beta \in(0,1]$. Assume $\alpha \in\left[0, y_{1} \beta\right)$, where $y_{1}$ is a certain constant from the interval $(0,1)$ (see Lemma 3.4 and Appendix B). Let $a \in\left(C^{(1)}(\bar{\Omega})\right)^{3}$ satisfy one of the conditions i), ii), iii) given by Theorem 3.9. Then, for all $f \in L_{\alpha+1, \beta}^{2}(\Omega)$, there exists a unique weak solution $u \in V_{\alpha, \beta}(\Omega)$ to problem (1.1)-(1.3) with

$$
\|u\|_{2, \alpha-1, \beta}^{2}+\|\nabla u\|_{2, \alpha, \beta}^{2} \leq C\|f\|_{2, \alpha+1, \beta}^{2}
$$

for some positive constant $C$.

\section{Friedrichs-Poincaré inequality}

In this section we derive some inequality of the Friedrichs-Poincare type in weighted Sobolev spaces. 
Proposition 2.1. For arbitrary $\alpha, \beta \geq 0$ we have

$$
\Delta \eta_{\beta}^{\alpha}(x) \geq 2 \beta \min (1, \beta) \varepsilon \delta \eta_{\beta-1}^{\alpha-1}(x)
$$

for all $x \in \boldsymbol{R}^{3}, x \neq 0$.

Proof. We introduce $\beta^{*}=\min (\beta, 1)$ in $(1.8)$ for $r>0$ i.e. $x \neq 0$ :

$$
\begin{aligned}
\Delta \eta_{\beta}^{\alpha}=\{ & \left(\alpha^{2} \delta^{2} \frac{1+\varepsilon s}{1+\delta r}-\alpha \delta^{2} \frac{1+\varepsilon s}{1+\delta r}\right)+2 \alpha \beta \delta \varepsilon \frac{s}{r}+2 \beta(\beta-1) \frac{\varepsilon}{r}(1+\delta r) \frac{\varepsilon s}{1+\varepsilon s} \\
& \left.+2 \alpha \delta^{2}(1+\varepsilon s) \frac{1}{\delta r}+\left(1-\beta^{*}+\beta^{*}\right) 2 \beta \frac{\varepsilon}{r}(1+\delta r)\right\} \eta_{\beta-1}^{\alpha-1},
\end{aligned}
$$

for $r>0$. We denote the five terms in \{\} by $T_{1}, T_{2}, \ldots, T_{5}$, and overwrite the previous relation as

$$
\Delta \eta_{\beta}^{\alpha}=\left\{\left[T_{1}+T_{4}\right]+T_{2}+\left[T_{3}+\left(1-\beta^{*}\right) T_{5}\right]+\beta^{*} T_{5}\right\} \eta_{\beta-1}^{\alpha-1}
$$

Observing that $T_{5} \geq 2 \beta \varepsilon \delta$, the proposition is trivial.

Proposition 2.2. Let $\alpha \geq 0, \beta \geq 0, \delta>0, \varepsilon>0$ and $\kappa>1$. Then:

$$
\left|\nabla \eta_{\beta}^{\alpha}(x)\right|^{2} \leq 2 \kappa \delta \varepsilon(\alpha+\beta)^{2}\left(\eta_{\beta-1 / 2}^{\alpha-1 / 2}(x)\right)^{2}
$$

for $x \in \boldsymbol{R}^{3},|x| \geq|1 / \delta-1 /(2 \varepsilon)| /(\kappa-1)$.

Let $\alpha \geq 0, \beta \geq 0$, and $\delta$ and $\varepsilon$ be arbitrary positive constants, such that $(\beta-\alpha)(2 \varepsilon-\delta) \geq 0$. Then:

$$
\left|\nabla \eta_{\beta}^{\alpha}(x)\right|^{2} \leq(\alpha \delta+2 \beta \varepsilon)^{2}\left(\eta_{\beta-1 / 2}^{\alpha-1 / 2}(x)\right)^{2}
$$

for $x \in \boldsymbol{R}^{3}, x \neq 0$.

Proof. If $\beta=0$ and $\alpha=0$ then the assertions a) and b) are valid. Let us concentrate on the nontrivial case:

a) From (1.7) for $r>0, s \in[0,2 r]$, we have

$$
\begin{aligned}
\left|\nabla \eta_{\beta}^{\alpha}(x)\right|^{2} & =g(s(x), r(x))\left(\eta_{\beta-1 / 2}^{\alpha-1 / 2}(x)\right)^{2} \\
g(s, r) & \equiv\left\{\alpha^{2} \delta^{2}\left(\frac{1+\varepsilon s}{1+\delta r}\right)+2 \alpha \beta \delta \varepsilon \frac{s}{r}+2 \beta^{2} \varepsilon^{2}\left(\frac{1+\delta r}{1+\varepsilon s}\right) \frac{s}{r}\right\} \\
\frac{\partial g}{\partial s}(s, r) & >0 \quad \text { for } s \in[0,2 r] .
\end{aligned}
$$

So, $g(s, r)$ is increasing as a function of $s$ and 


$$
\begin{aligned}
G(r) & \equiv \max _{s \in[0,2 r]} g(s, r)=g(2 r, r)=\alpha^{2} \delta^{2} \frac{1+2 \varepsilon r}{1+\delta r}+4 \alpha \beta \delta \varepsilon+4 \beta^{2} \varepsilon^{2} \frac{1+\delta r}{1+2 \varepsilon r} \\
& \leq 2 \kappa(\alpha+\beta)^{2} \delta \varepsilon
\end{aligned}
$$

for $\kappa>1$ and $r \geq|1 / \delta-1 /(2 \varepsilon)| /(\kappa-1)$. So, inequality a) is proved.

b) To justify second inequality $b$ ), we observe that for the given values of $\alpha, \beta, \delta, \varepsilon$ and for $r>0, G(r) \leq G(0)$.

Next we derive an inequality of the Friedrichs-Poincaré type in the space $\stackrel{\circ}{H}_{\alpha, \beta}^{1}(\Omega)$. It is necessary for our aim to get expressions of constants in this inequality. Let us recall that $\stackrel{\circ}{H}_{\alpha, \beta}^{1}(\Omega)$ is a completion of $C_{0}^{\infty}(\Omega)$ in the norm

$$
v \mapsto \int_{\Omega} v^{2} \eta_{\beta-1}^{\alpha-1} d x+\int_{\Omega}|\nabla v|^{2} \eta_{\beta}^{\alpha} d x
$$

It follows from Proposition 2.1

Lemma 2.3. Let $\alpha \geq 0, \beta>0, \alpha+\beta<3, \kappa>1$. Let $\delta$ and $\varepsilon$ be arbitrary positive constants, such that $(\beta-\alpha)(2 \varepsilon-\delta) \geq 0$. Then for all $u \in \stackrel{\circ}{H}_{\alpha, \beta}^{1}(\Omega)$

$$
\begin{aligned}
\beta \beta^{*} \delta \varepsilon \int_{\Omega} u^{2} \eta_{\beta-1}^{\alpha-1} d x \leq & \frac{(\alpha \delta+2 \beta \varepsilon)^{2}}{\beta \beta^{*} \delta \varepsilon} \int_{\Omega_{R_{0}}}|\nabla u|^{2} \eta_{\beta}^{\alpha} d x \\
& +\frac{2 \kappa(\alpha+\beta)^{2}}{\beta \beta^{*}} \int_{\Omega^{R_{0}}}|\nabla u|^{2} \eta_{\beta}^{\alpha} d x
\end{aligned}
$$

where $R_{0} \geq|1 / \delta-1 /(2 \varepsilon)| /(\kappa-1)$. Moreover, if $\delta=2 \varepsilon$ then

$$
\|u\|_{2, \alpha-1, \beta-1} \leq\left(\frac{\alpha+\beta}{\beta \beta^{*} \varepsilon}\right)\|\nabla u\|_{2, \alpha, \beta} .
$$

Proof. Due to the density of $C_{0}^{\infty}(\Omega)$ in $\stackrel{\circ}{H}_{\alpha, \beta}^{1}(\Omega)$ it is sufficient to prove the inequality for all $u \in C_{0}^{\infty}(\Omega)$. From Proposition 2.1 it follows that for $v \in C_{0}^{\infty}(\Omega)$

$$
\begin{aligned}
2 \beta \beta^{*} \delta \varepsilon \int_{\Omega} v^{2} \eta_{\beta-1}^{\alpha-1} d x & \leq \int_{\Omega} v^{2} \Delta \eta_{\beta}^{\alpha} d x=-2 \int_{\Omega} v \nabla v \cdot \nabla \eta_{\beta}^{\alpha} d x \\
& \leq \beta \beta^{*} \delta \varepsilon \int_{\Omega}|v|^{2} \eta_{\beta-1}^{\alpha-1} d x+\frac{1}{\beta \beta^{*} \delta \varepsilon} \int_{\Omega}|\nabla v|^{2}\left|\nabla \eta_{\beta}^{\alpha}\right|^{2} \frac{1}{\eta_{\beta-1}^{\alpha-1}} d x
\end{aligned}
$$

hence, we have:

$$
\beta \beta^{*} \delta \varepsilon \int_{\Omega} v^{2} \eta_{\beta-1}^{\alpha-1} d x \leq \frac{1}{\beta \beta^{*} \delta \varepsilon} \int_{\Omega}|\nabla v|^{2} \frac{\left|\nabla \eta_{\beta}^{\alpha}\right|^{2}}{\eta_{\beta-1}^{\alpha-1}} d x
$$


From Proposition 2.2 with $\Omega=\Omega_{R_{0}} \cup \Omega^{R_{0}}, R_{0} \geq|1 / \delta-1 /(2 \varepsilon)| /(\kappa-1)$ we get:

$$
\begin{aligned}
\beta \beta^{*} \delta \varepsilon \int_{\Omega} v^{2} \eta_{\beta-1}^{\alpha-1} d x \leq & \frac{(\alpha \delta+2 \beta \varepsilon)^{2}}{\beta \beta^{*} \delta \varepsilon} \int_{\Omega_{R_{0}}}|\nabla v|^{2} \eta_{\beta}^{\alpha} d x \\
& +\frac{2 \kappa(\alpha+\beta)^{2}}{\beta \beta^{*}} \int_{\Omega^{R_{0}}}|\nabla v|^{2} \eta_{\beta}^{\alpha} d x
\end{aligned}
$$

In particular, if $\delta=2 \varepsilon$ it follows from this relation the inequality $\|u\|_{2, \alpha-1, \beta-1} \leq$ $\kappa^{1 / 2}\left((\alpha+\beta) /\left(\beta \beta^{*} \varepsilon\right)\right)\|\nabla u\|_{2, \alpha, \beta}$ for all $\kappa>1$. So, we get the relation (2.14).

Remark 2.4. In the next we will use Lemma 2.3 in the form given by Theorem 1.4 in Introduction, i.e. (1.11) with

$$
c_{0}=\left(\frac{\alpha \delta+2 \beta \varepsilon}{\beta \beta^{*} \delta \varepsilon}\right)^{2}, \quad c_{1}=\frac{2 \kappa}{\delta \varepsilon}\left(\frac{\alpha+\beta}{\beta \beta^{*}}\right)^{2} .
$$

Let us observe that if additionally $\delta<2 \varepsilon$ and $1<\kappa \leq(2 \varepsilon) / \delta+\delta /(2 \varepsilon)-1$ then $c_{0} \geq c_{1}$.

Remark 2.5. The usefulness of $\beta>0$ arises not only from possibility to justify the previous weighted Friedrichs-Poincaré type inequality, but also from the properties of $\partial_{1} \eta_{\beta}^{\alpha}$.

Remark 2.6. From the Hardy's inequality, one can derive

$$
\|u\|_{2,-2, \beta} \leq c\|\nabla u\|_{2,0, \beta}
$$

for $\beta>0$, i.e. another Poincaré type inequality which will be of interest to prove the coercivity in Section 3.3, for "small" $\operatorname{div}\left(a \eta_{\beta}^{\alpha}\right)$.

Let us recall the Hardy's inequality we will use (for the proof see [9], p. 33):

Proposition 2.7. Let $1<p<\infty, \epsilon \neq p-1$. Let $u=u(t)$ be a function differentiable a.e. in $(0, \infty)$ such that

$$
\begin{gathered}
\int_{0}^{\infty}\left|u^{\prime}(t)\right|^{p} t^{\epsilon} d t<\infty, \\
\lim _{t \rightarrow 0+} u(t)=0 \quad \text { for } \epsilon<p-1, \quad \lim _{t \rightarrow+\infty} u(t)=0 \quad \text { for } \epsilon>p-1 .
\end{gathered}
$$

Then the following inequality is satisfied:

$$
\int_{0}^{\infty}|u(t)|^{p} t^{\epsilon-p} d t \leq\left(\frac{p}{\epsilon-p+1}\right)^{p} \int_{0}^{\infty}\left|u^{\prime}(t)\right|^{p} t^{\epsilon} d t .
$$

For an arbitrary function $\phi \in C_{0}^{\infty}(\Omega)$ we will use in the proof of the next lemma the same notation for the extension of the function by zero on $\boldsymbol{R}^{3}$. 
Because $0 \notin \operatorname{supp} \phi$ (see section 1.2), $\phi \equiv 0$ in some neighbourhood of point 0 . We obtain:

Lemma 2.8. For $\beta>0$ and an arbitrary function $\phi \in C_{0}^{\infty}(\Omega)$ the following relation is satisfied:

$$
\int_{\Omega} \phi^{2} \frac{1}{|x|^{2}} \eta_{\beta}^{0} d x \leq 2^{2+|1-\beta|} \int_{\Omega}|\nabla \phi|^{2} \eta_{\beta}^{0} d x .
$$

Proof. Let us denote $\Phi(r, \varphi, \psi) \equiv \phi(r \sin \psi, r \cos \varphi \cos \psi, r \sin \varphi \cos \psi)$, $r \in[0, \infty), \varphi \in[0,2 \pi), \psi \in[-\pi / 2, \pi / 2]$. Let $p=2, \epsilon=2+\beta, \beta>-1$. From Hardy's inequality it follows

$$
\int_{0}^{\infty} \Phi^{2} r^{\beta} d r \leq \frac{4}{(1+\beta)^{2}} \int_{0}^{\infty}\left(\frac{d \Phi}{d r}\right)^{2} r^{2+\beta} d r
$$

and after integration with respect to $\varphi$ and $\psi$ we have

$$
\begin{aligned}
& \int_{0}^{2 \pi}\left[\int_{-\pi / 2}^{\pi / 2}\left(\int_{0}^{\infty} \Phi^{2} r^{\beta} d r\right)(1-\sin \psi)^{\beta} \cos \psi d \psi\right] d \varphi \\
& \quad \leq \frac{4}{(1+\beta)^{2}} \int_{0}^{2 \pi}\left[\int_{-\pi / 2}^{\pi / 2}\left(\int_{0}^{\infty}\left(\frac{d \Phi}{d r}\right)^{2} r^{2+\beta} d r\right)(1-\sin \psi)^{\beta} \cos \psi d \psi\right] d \varphi .
\end{aligned}
$$

So, in Cartesian coordinates we get:

$$
\int_{\Omega} \phi^{2} \frac{1}{|x|^{2}} s^{\beta} d x \leq \frac{4}{(1+\beta)^{2}} \int_{\Omega}\left|\nabla \phi \cdot \frac{x}{|x|}\right|^{2} s^{\beta} d x \leq \frac{4}{(1+\beta)^{2}} \int_{\Omega}|\nabla \phi|^{2} s^{\beta} d x .
$$

Repeating the consideration for $\beta=0$ and using elementary inequality (1.4) we get the assertion of the lemma.

\section{A model equation with non-constant coefficient functions}

\subsection{The model problem in $\boldsymbol{\Omega}_{R}$}

We will study in this section the existence of a weak solution of the problem (1.1), (1.2) in a bounded domain $\Omega_{R}$. We will need some technical lemmas.

Let us define a function $F_{\alpha, \beta}(s, r ; v)$ by the relation:

$$
F_{\alpha, \beta}(s, r ; v) \eta_{\beta-1}^{\alpha-1} \equiv-v \frac{\left|\nabla \eta_{\beta}^{\alpha}\right|^{2}}{\eta_{\beta}^{\alpha}}-k \partial_{1} \eta_{\beta}^{\alpha}
$$

The following lemma gives the evaluation of $F_{\alpha, \beta}(s, r ; v)$ from below. 
Lemma 3.1. Let $0 \leq \alpha<\beta, \kappa>1, \quad 0<\varepsilon \leq(1 /(2 \kappa)) \cdot(k / v) \cdot\left((\beta-\alpha) / \beta^{2}\right)$ and $\delta, v, k>0$. Then

$$
F_{\alpha, \beta}(s, r ; v)-\left(1-\frac{1}{\kappa}\right) k \delta \varepsilon(\beta-\alpha) s \geq-\alpha \delta k\left(1+\frac{v}{k} \alpha \delta\right)
$$

for all $r>0$ and $s \in[0,2 r]$.

Proof. Expressing the function $F_{\alpha, \beta}(s, r ; v)$ explicitly we get

$$
\begin{aligned}
F_{\alpha, \beta}(s, r ; v)= & -v \alpha^{2} \delta^{2}\left(\frac{1+\varepsilon s}{1+\delta r}\right)-2 v \alpha \beta \delta \varepsilon \frac{s}{r}-2 v \beta^{2} \varepsilon^{2}\left(\frac{1+\delta r}{1+\varepsilon s}\right) \frac{s}{r} \\
& -k \alpha \delta(1+\varepsilon s) \frac{r-s}{r}+k \beta \varepsilon(1+\delta r) \frac{s}{r} .
\end{aligned}
$$

For convenient use we subtract $(1-1 / \kappa) k \delta \varepsilon(\beta-\alpha) s$ from $F_{\alpha, \beta}(s, r ; v)$. We observe (see Appendix A) that, for the given $\alpha, \beta, \varepsilon, \kappa$, for all $\delta, v, k>0$ and for $r>0$,

$$
F_{\alpha, \beta}(s, r ; v)-\left(1-\frac{1}{\kappa}\right) k \delta \varepsilon(\beta-\alpha) s \geq F_{\alpha, \beta}(0, r ; v),
$$

which immediately gives inequality (3.17).

Let us recall the model problem on $\Omega_{R}$ :

$$
\begin{gathered}
-v \Delta u+k \partial_{1} u+a \cdot \nabla u=f \quad \text { in } \Omega_{R}, \\
u=0 \quad \text { on } \partial \Omega_{R} \equiv \partial \Omega_{c} \cup \partial B_{R} .
\end{gathered}
$$

We show the existence of a weak solution $u_{R} \in \stackrel{\circ}{H}^{1}\left(\Omega_{R}\right)$ of this problem. Following (1.9), (1.10) again with $w=\eta_{\beta_{0}}^{0}, \beta_{0} \in(0,1]$, using notation (3.16), let us introduce a continuous bilinear form $Q_{1}(\cdot, \cdot)$ on $\stackrel{\circ}{H}^{1}\left(\Omega_{R}\right) \times \stackrel{\circ}{H^{1}}\left(\Omega_{R}\right)$ :

$$
\begin{gathered}
Q_{1}(u, v)=\int_{\Omega_{R}} v \nabla u \cdot \nabla\left(v \eta_{\beta_{0}}^{0}\right) d x+k \int_{\Omega_{R}} \partial_{1} u\left(v \eta_{\beta_{0}}^{0}\right) d x \\
\quad+\int_{\Omega_{R}}(a \cdot \nabla u)\left(v \eta_{\beta_{0}}^{0}\right) d x \\
Q_{1}(v, v) \geq \frac{v}{2} \int_{\Omega_{R}}|\nabla v|^{2} \eta_{\beta_{0}}^{0} d x+\frac{1}{2} \int_{\Omega_{R}} v^{2} F_{0, \beta_{0}}(s, r ; v) \eta_{\beta_{0}-1}^{-1} d x \\
\quad-\frac{1}{2} \int_{\Omega_{R}} v^{2} \operatorname{div}\left(\eta_{\beta_{0}}^{0} a\right) d x .
\end{gathered}
$$

We need a certain smallness of the positive part of $\operatorname{div}\left(\eta_{\beta_{0}}^{0} a\right)$. We will ask for estimate of this term from above in the following form with appropriate constants $C_{1}, C_{2}, C_{3}>0: \quad \operatorname{div}\left(\eta_{\beta_{0}}^{0} a\right) \leq C_{1} \eta_{\beta_{0}-1}^{-1}+C_{2} \eta_{\beta_{0}}^{0} /|x|^{2}+C_{3}\left|\nabla \eta_{\beta_{0}}^{0}\right|^{2} / \eta_{\beta_{0}}^{0}$. The 
corresponding integrals to the first two terms could be estimated using (2.13) and (2.15) respectively. The third term can be covered by the expression $F_{0, \beta_{0}}(s, r ; v)$ : for $\alpha=0$ we have from Lemma 3.1 that $F_{0, \beta_{0}}(s, r ; v) \eta_{\beta_{0}-1}^{-1}$ $C_{3}\left|\nabla \eta_{\beta_{0}}^{0}\right|^{2} / \eta_{\beta_{0}}^{0} \equiv F_{0, \beta_{0}}\left(s, r ; v+C_{3}\right) \eta_{\beta_{0}-1}^{-1} \geq 0$. So, let us make the following assumption on $a$ :

$$
\begin{aligned}
\operatorname{div}\left(\eta_{\beta_{0}}^{0} a\right) \leq & A \frac{v_{0}\left(\beta_{0}^{*}\right)^{2} \varepsilon_{0}^{2}}{4} \eta_{\beta_{0}-1}^{-1}+(1-A) \frac{v_{0}}{2^{3-\beta_{0}}} \frac{\eta_{\beta_{0}}^{0}}{r^{2}} \\
& +v(N-1) \frac{\left|\nabla \eta_{\beta_{0}}^{0}\right|^{2}}{\eta_{\beta_{0}}^{0}}
\end{aligned}
$$

for some $\quad N>1, \quad 0 \leq A \leq 1, \quad 0<v_{0}<v, \quad \varepsilon_{0}<(1 / 2) \cdot(k /(v N)) \cdot\left(1 / \beta_{0}\right)$, $\eta_{\beta_{0}}^{\alpha} \equiv \eta_{\beta_{0}, \varepsilon_{0}}^{\alpha, \varepsilon_{0}}$.

Lemma 3.2. Let $0<\beta_{0} \leq 1$ and $a \in\left(C^{(1)}\left(\bar{\Omega}_{R}\right)\right)^{3}$ satisfying condition (3.21). Then, for all $f \in L_{1, \beta_{0}}^{2}\left(\Omega_{R}\right)$, there exists $u_{R} \in \stackrel{\circ}{H}^{1}\left(\Omega_{R}\right)$, the unique solution of

$$
Q_{1}\left(u_{R}, v\right)=\int_{\Omega_{R}} f v \eta_{\beta_{0}}^{0} d x
$$

for all $v \in \stackrel{\circ}{H}^{1}\left(\Omega_{R}\right)$.

Proof. There exists a constant of coercivity $C=C(R)>0$ such that

$$
Q_{1}(v, v) \geq C\|v\|^{2},
$$

where $\|\cdot\|$ is here the norm in the space $\stackrel{\circ}{H}^{1}\left(\Omega_{R}\right)$. Indeed, using condition (3.21) we have from $(3.20)(\kappa>1)$ :

$$
\begin{aligned}
Q_{1}(v, v) \geq & E\left(v, \Omega_{R}\right) \equiv\left(1-\frac{1}{\kappa}\right) \frac{v}{2} \int_{\Omega_{R}}|\nabla v|^{2} \eta_{\beta_{0}}^{0} d x \\
& +\frac{A}{2}\left(\frac{v}{\kappa} \int_{\Omega_{R}}|\nabla v|^{2} \eta_{\beta_{0}}^{0} d x-\int_{\Omega_{R}} v^{2} \frac{v_{0}\left(\beta_{0}^{*}\right)^{2} \varepsilon_{0}^{2}}{4} \eta_{\beta_{0}-1}^{-1} d x\right) \\
& +\frac{1-A}{2}\left(\frac{v}{\kappa} \int_{\Omega_{R}}|\nabla v|^{2} \eta_{\beta_{0}}^{0} d x-\int_{\Omega_{R}} v^{2} \frac{v_{0}}{2^{3-\beta_{0}}} \frac{\eta_{\beta_{0}}^{0}}{|x|^{2}} d x\right) \\
& +\frac{1}{2} \int_{\Omega_{R}} v^{2}\left(F_{0, \beta_{0}}(s, r ; v) \eta_{\beta_{0}-1}^{-1}-v(N-1) \frac{\left|\nabla \eta_{\beta_{0}}^{0}\right|^{2}}{\eta_{\beta_{0}}^{0}}\right) d x .
\end{aligned}
$$

If $1<\kappa<v / v_{0}, \delta=\varepsilon_{0}, 1<\kappa<3 / 2$, we have from Remark 2.4:

$$
\int_{\Omega_{R}} v^{2} \frac{v_{0}\left(\beta_{0}^{*}\right)^{2} \varepsilon_{0}^{2}}{4} \eta_{\beta_{0}-1}^{-1} d x \leq \frac{v}{\kappa} \int_{\Omega_{R}}|\nabla v|^{2} \eta_{\beta_{0}}^{0} d x .
$$


If $1<\kappa<v / v_{0}$, it follows from Lemma 2.8:

$$
\int_{\Omega_{R}} v^{2} \frac{v_{0}}{2^{3-\beta_{0}}} \frac{1}{|x|^{2}} \eta_{\beta_{0}}^{0} d x \leq \frac{v}{\kappa} \int_{\Omega_{R}}|\nabla v|^{2} \eta_{\beta_{0}}^{0} d x .
$$

Because $\varepsilon_{0}<(1 / 2) \cdot(k /(v N)) \cdot\left(1 / \beta_{0}\right)$ there is a constant $\kappa$ satisfying all previous conditions and additionally $\varepsilon_{0} \leq(1 /(2 \kappa)) \cdot(k /(v N)) \cdot\left(1 / \beta_{0}\right)$. We get:

$$
\begin{aligned}
\int_{\Omega_{R}} & v^{2}\left(F_{0, \beta_{0}}(s, r ; v) \eta_{\beta_{0}-1}^{-1}-v(N-1) \frac{\left|\nabla \eta_{\beta_{0}}^{0}\right|^{2}}{\eta_{\beta_{0}}^{0}}\right) d x \\
& \equiv \int_{\Omega_{R}} v^{2} F_{0, \beta_{0}}(s, r ; v N) \eta_{\beta_{0}-1}^{-1} d x \geq\left(1-\frac{1}{\kappa}\right) k \varepsilon_{0}^{2} \beta_{0} \int_{\Omega_{R}} v^{2} \eta_{\beta_{0}-1}^{-1} s d x .
\end{aligned}
$$

From the relations (3.24)-(3.27) we get

$$
Q_{1}(v, v) \geq\left(1-\frac{1}{\kappa}\right)\left[\frac{v}{2} \int_{\Omega_{R}}|\nabla v|^{2} \eta_{\beta_{0}}^{0} d x+\frac{k \varepsilon_{0} \beta_{0}}{2} \int_{\Omega_{R}} v^{2} \eta_{\beta_{0}-1}^{-1}\left(\varepsilon_{0} s\right) d x\right] .
$$

Using Lemma 2.3 and Remark 2.4 we derive:

$$
\begin{gathered}
Q_{1}(v, v) \geq\left(1-\frac{1}{\kappa}\right)\left[\frac{v}{4} \int_{\Omega_{R}}|\nabla v|^{2} \eta_{\beta_{0}}^{0} d x+\frac{v \varepsilon_{0}^{2} \beta_{0}^{2}}{16} \int_{\Omega_{R}} v^{2} \eta_{\beta_{0}-1}^{-1} d x\right. \\
\left.+\frac{k \varepsilon_{0} \beta_{0}}{2} \int_{\Omega_{R}} v^{2} \eta_{\beta_{0}-1}^{-1}\left(\varepsilon_{0} s\right) d x\right] .
\end{gathered}
$$

The sum of the second and the third integrals can be estimated by an integral of $v^{2} \eta_{\beta_{0}}^{-1}$, because $\eta_{\beta_{0}-1}^{-1}+\eta_{\beta_{0}-1}^{-1}\left(\varepsilon_{0} s\right)=\eta_{\beta_{0}-1}^{-1}\left(1+\varepsilon_{0} s\right)=\eta_{\beta_{0}}^{-1}$ :

$$
\begin{array}{r}
Q_{1}(v, v) \geq\left(1-\frac{1}{\kappa}\right) \frac{v}{4} \min \left\{1, \frac{1}{4} \varepsilon_{0}^{2} \beta_{0}^{2}, \frac{2 k}{v} \beta_{0} \varepsilon_{0}\right\} \\
\cdot\left(\int_{\Omega_{R}}|\nabla v|^{2} \eta_{\beta_{0}}^{0} d x+\int_{\Omega_{R}} v^{2} \eta_{\beta_{0}}^{-1} d x\right)
\end{array}
$$

Hence, we get (3.23) with $C=(1-1 / \kappa) v / 4 \min \left\{1, \varepsilon_{0}^{2} \beta_{0}^{2} / 4,2 k \beta_{0} \varepsilon_{0} / v\right\}\left(1+\varepsilon_{0} R\right)^{-1}$, and the inequality (3.23) is proved. Using Lax-Milgram theorem we get that there is $u_{R} \in \stackrel{\circ}{H}^{1}\left(\Omega_{R}\right)$ such that (3.22) is satisfied.

Remark 3.3. An arbitrary function $\Phi \in \stackrel{\circ}{H}^{1}\left(\Omega_{R}\right)$ can be expressed in the form $\phi \eta_{\beta_{0}}^{0}$, where $\phi$ is a function from $\stackrel{\circ}{H}^{1}\left(\Omega_{R}\right)$. Therefore we have also for $u_{R}$

$$
Q\left(u_{R}, \Phi\right)=\int_{\Omega_{R}} f \Phi d x
$$

for an all $\Phi \in \stackrel{\circ}{H}^{1}\left(\Omega_{R}\right)$, where by the definition $Q\left(u_{R}, \Phi\right) \equiv Q\left(u_{R}, \phi \eta_{\beta_{0}}^{0}\right) \equiv$ $Q_{1}(u, \phi)$. 


\subsection{Uniform estimates of $u_{R}$}

Our next aim is to prove that the weak solutions $u_{R}$ of (3.22) are uniformly bounded in $V_{\alpha, \beta}(\Omega)$ as $R \rightarrow+\infty$. To prove this, we need some additional asymptotic property of function $a$ : Let $\gamma=\gamma(\rho)$ be a nonnegative nonincreasing continuous function defined on interval $[m,+\infty)$ with the limit $\lim _{\rho \rightarrow+\infty} \gamma(\rho)=0$, and the function $a$ satisfies the following condition:

$$
\operatorname{div}\left(\eta_{\beta}^{\alpha}(x) a(x)\right) \leq \gamma(|x|) \eta_{\beta-1}^{\alpha-1}(x),
$$

for all $x \in \Omega\left(\eta_{\beta}^{\alpha} \equiv \eta_{\beta, \varepsilon}^{\alpha, \delta}, \delta>0, \varepsilon>0\right)$.

Let $y_{1}$ be the unique real solution of the algebraic equation $4 y^{3}+8 y^{2}+$ $5 y-1=0$. It is easy to verify that $y_{1} \in(0,1)$. We will also explain later, why the control of $\alpha / \beta$ by $y_{1}$ is necessary.

Lemma 3.4. Let $0<\beta \leq 1,0 \leq \alpha<y_{1} \beta, f \in L_{\alpha+1, \beta}^{2}(\Omega)$ and $a \in\left(C^{(1)}(\bar{\Omega})\right)^{3}$ such that conditions (3.21), (3.30) are satisfied. Then, as $R \rightarrow+\infty$, the weak solutions $u_{R}$ of (3.22) given by Lemma 3.2 are uniformly bounded in $V_{\alpha, \beta}(\Omega)$. There is a constant $C>0$, which does not depend on $R$, such that

$$
\int_{\Omega} \tilde{u}_{R}^{2} \eta_{\beta}^{\alpha-1} d x+\int_{\Omega}\left|\nabla \tilde{u}_{R}\right|^{2} \eta_{\beta}^{\alpha} d x \leq C \int_{\Omega}|f|^{2} \eta_{\beta}^{\alpha+1} d x
$$

for all $R$ greater than some $R_{0}>0, \tilde{u}_{R}$ being extension by zero of $u_{R}$ on $\Omega \backslash \Omega_{R}$.

Proof. First, we derive estimate of $u_{R}$ on a bounded subdomain $\Omega_{R_{0}} \subset \Omega_{R}$, where $0<M<R_{0}<R$; The choice of $R_{0}$ will be given in the next part of the proof. Our aim is to get an estimate with a constant not depending on $R$. Let us substitute $\phi=u_{R}$ into (3.22). Hence, it follows from (3.28):

$$
C_{1}\left(\int_{\Omega_{R}}\left|\nabla u_{R}\right|^{2} \eta_{\beta_{0}}^{0} d x+\int_{\Omega_{R}} u_{R}^{2} \eta_{\beta_{0}}^{-1} d x\right) \leq Q_{1}\left(u_{R}, u_{R}\right)=\int_{\Omega_{R}} f u_{R} \eta_{\beta_{0}}^{0} d x,
$$

with the constant $C_{1}>0$ stated in (3.28). Let $R_{0}$ be some fixed positive number such that $0<M<R_{0}<R$. We get

$$
\int_{\Omega_{R_{0}}}\left|\nabla u_{R}\right|^{2} \eta_{\beta}^{\alpha} d x+\int_{\Omega_{R_{0}}} u_{R}^{2} \eta_{\beta}^{\alpha-1} d x \leq C_{2} \int_{\Omega_{R}}|f|\left|u_{R}\right| \eta_{\beta}^{\alpha} d x,
$$

where the constant $C_{2}=C_{1}^{-1}\left(1+\varepsilon_{0} R_{0}\right)^{\alpha}\left(1+2 \varepsilon_{0} R_{0}\right)^{\left|\beta-\beta_{0}\right|}$ depend on $k, v, \alpha, \beta$, $\beta_{0}, \varepsilon_{0}, R_{0}, \kappa$, but does not depend on $R$.

Now, we are going to derive an estimate of $u_{R}$ on domain $\Omega_{R}$. Using the test function $\Phi=u_{R} \eta_{\beta}^{\alpha}=u_{R}(1+\delta r)^{\alpha}(1+\varepsilon s)^{\beta} \in \stackrel{\circ}{H}^{1}\left(\Omega_{R}\right)$ in (3.29) we get after integration by parts: 


$$
\begin{gathered}
v \int_{\Omega_{R}}\left|\nabla u_{R}\right|^{2} \eta_{\beta}^{\alpha} d x+v \int_{\Omega_{R}} u_{R} \nabla u_{R} \cdot \nabla \eta_{\beta}^{\alpha} d x-\frac{k}{2} \int_{\Omega_{R}} u_{R}^{2} \partial_{1} \eta_{\beta}^{\alpha} d x \\
\quad-\frac{1}{2} \int_{\Omega_{R}} \operatorname{div}\left(\eta_{\beta}^{\alpha} a\right) u_{R}^{2} d x=\int_{\Omega_{R}} f u_{R} \eta_{\beta}^{\alpha} d x .
\end{gathered}
$$

So, taking condition (3.30) into account we get for some $\kappa>1$ :

$$
\begin{aligned}
& \frac{v}{2 \kappa} \int_{\Omega_{R}}\left|\nabla u_{R}\right|^{2} \eta_{\beta}^{\alpha} d x \\
&+ \frac{1}{2}\left[v\left(1-\frac{1}{\kappa}\right) \int_{\Omega_{R}}\left|\nabla u_{R}\right|^{2} \eta_{\beta}^{\alpha} d x-\int_{\Omega_{R}} u_{R}^{2} \gamma(|x|) \eta_{\beta-1}^{\alpha-1} d x\right] \\
& \quad+\frac{1}{2} \int_{\Omega_{R}} u_{R}^{2} F_{\alpha, \beta}(s, r ; v) \eta_{\beta-1}^{\alpha-1} d x \leq \int_{\Omega_{R}}|f|\left|u_{R}\right| \eta_{\beta}^{\alpha} d x .
\end{aligned}
$$

Let $R_{0}$ be a positive constant such that

$$
R_{0} \geq\left|\frac{1}{\delta}-\frac{1}{2 \varepsilon}\right| \frac{1}{(\kappa-1)} \quad \text { and } \quad \gamma\left(R_{0}\right)<\frac{v \delta \varepsilon}{2} \frac{\kappa-1}{\kappa^{2}}\left(\frac{\beta \beta^{*}}{\alpha+\beta}\right)^{2}
$$

We will use the notation $\Omega_{R}^{R_{0}}=\Omega^{R_{0}} \cap \Omega_{R}$ assuming $R>R_{0}$. We are going to estimate the left hand side in (3.33) from below. For our aim it is only necessary to express coefficients of integrals taken over $\Omega_{R}^{R_{0}}$, while coefficients of integrals over $\Omega_{R_{0}}$ we will denote by $C_{i}>0, i=1,2, \ldots$, verifying only that these constants do not depend on $R$.

Using Lemma 2.3 and relation (3.34) we have:

$$
\begin{aligned}
\int_{\Omega_{R}} \gamma(|x|) u_{R}^{2} \eta_{\beta-1}^{\alpha-1} d x \leq & \int_{\Omega_{R_{0}}} \gamma(|x|) u_{R}^{2} \eta_{\beta-1}^{\alpha-1} d x+\int_{\Omega_{R}^{R_{0}}} \gamma\left(R_{0}\right) u_{R}^{2} \eta_{\beta-1}^{\alpha-1} d x \\
\leq & 2 C_{3}\left(\int_{\Omega_{R_{0}}} u_{R}^{2} \eta_{\beta-1}^{\alpha-1} d x+\int_{\Omega_{R_{0}}}\left|\nabla u_{R}\right|^{2} \eta_{\beta}^{\alpha} d x\right) \\
& +\frac{2 \gamma\left(R_{0}\right) \kappa}{\delta \varepsilon}\left(\frac{\alpha+\beta}{\beta \beta^{*}}\right)^{2} \int_{\Omega_{R}^{R_{0}}}\left|\nabla u_{R}\right|^{2} \eta_{\beta}^{\alpha} d x
\end{aligned}
$$

Due to the choice of $R_{0}$ we have $(1-1 / \kappa) v / 2-\gamma\left(R_{0}\right) \kappa /(\delta \varepsilon) \cdot((\alpha+\beta) /$ $\left.\left(\beta \beta^{*}\right)\right)^{2} \geq 0$. So, the second term in (3.33) can be estimated

$$
\begin{gathered}
\frac{1}{2}\left[v\left(1-\frac{1}{\kappa}\right) \int_{\Omega_{R}}\left|\nabla u_{R}\right|^{2} \eta_{\beta}^{\alpha} d x-\int_{\Omega_{R}} u_{R}^{2} \gamma(|x|) \eta_{\beta-1}^{\alpha-1} d x\right] \\
\geq-C_{3}\left(\int_{\Omega_{R_{0}}} u_{R}^{2} \eta_{\beta-1}^{\alpha-1} d x+\int_{\Omega_{R_{0}}}\left|\nabla u_{R}\right|^{2} \eta_{\beta}^{\alpha} d x\right) .
\end{gathered}
$$


Using Lemma 3.1 (with $\left.0 \leq \alpha<\beta, \quad \varepsilon \leq(1 /(2 \kappa)) \cdot(k / v) \cdot\left((\beta-\alpha) / \beta^{2}\right)\right)$ and Lemma 2.3 (with $\delta<2 \varepsilon$ ), the third term in (3.33) can be estimated:

$$
\begin{aligned}
\int_{\Omega_{R}} u_{R}^{2} F_{\alpha, \beta}(s, r ; v) \eta_{\beta-1}^{\alpha-1} d x \\
\geq-\alpha \delta k\left(1+\frac{v \kappa}{k} \alpha \delta\right) \frac{2 \kappa}{\delta \varepsilon}\left(\frac{\alpha+\beta}{\beta \beta^{*}}\right)^{2} \int_{\Omega_{R}^{R_{0}}}\left|\nabla u_{R}\right|^{2} \eta_{\beta}^{\alpha} d x \\
\quad+\left(1-\frac{1}{\kappa}\right) k \delta \varepsilon(\beta-\alpha) \int_{\Omega_{R}^{R_{0}}} u_{R}^{2} \eta_{\beta-1}^{\alpha-1} s d x-2 C_{4} \int_{\Omega_{R_{0}}}\left|\nabla u_{R}\right|^{2} \eta_{\beta}^{\alpha} d x
\end{aligned}
$$

Denote $C_{5} \equiv(1 / 2) \alpha \delta k(1+\kappa \alpha \delta v / k)(2 \kappa /(\delta \varepsilon))\left((\alpha+\beta) /\left(\beta \beta^{*}\right)\right)^{2}$. It is clear that $C_{5} \leq v /\left(2 \kappa^{2}\right)<v /(2 \kappa)$ if $1+\kappa \alpha \delta v / k \leq \kappa$ (i.e. $\left.\delta \leq(k / v) \cdot((\kappa-1) /(\kappa \cdot \beta))\right)$ and $\alpha \leq\left(1 /\left(2 \kappa^{4}\right)\right) \cdot(v / k) \cdot\left(\left(\beta \beta^{*}\right) /(\alpha+\beta)\right)^{2} \varepsilon$. We have from $(3.33)$

$$
\begin{aligned}
& \frac{v}{2 \kappa}\left(1-\frac{1}{\kappa}\right) \int_{\Omega_{R}}\left|\nabla u_{R}\right|^{2} \eta_{\beta}^{\alpha} d x+\frac{1}{2}\left(1-\frac{1}{\kappa}\right) k \delta \varepsilon(\beta-\alpha) \int_{\Omega_{R}} u_{R}^{2} \eta_{\beta-1}^{\alpha-1} s d x \\
& \quad-C_{6} \int_{\Omega_{R_{0}}} u_{R}^{2} \eta_{\beta-1}^{\alpha-1} d x-C_{7} \int_{\Omega_{R_{0}}}\left|\nabla u_{R}\right|^{2} \eta_{\beta}^{\alpha} d x \leq \int_{\Omega_{R}}|f|\left|u_{R}\right| \eta_{\beta}^{\alpha} d x
\end{aligned}
$$

We overwrite the integrals computed on the domain $\Omega_{R_{0}}$ to the right hand side estimating them by inequality (3.32). Before using the mentioned inequality we should re-scale it with respect to new values $\varepsilon, \delta$, see Remark 1.2. The new constant in (3.32) after re-scaling we denote $C_{2}^{\prime}$. So, we get from the preceding inequality:

$$
\frac{v}{\kappa} \int_{\Omega_{R}}\left|\nabla u_{R}\right|^{2} \eta_{\beta}^{\alpha} d x+k \delta \varepsilon(\beta-\alpha) \int_{\Omega_{R}} u_{R}^{2} \eta_{\beta-1}^{\alpha-1} s d x \leq C_{8} \int_{\Omega_{R}}|f|\left|u_{R}\right| \eta_{\beta}^{\alpha} d x
$$

where $C_{8}=2\left\{1+C_{2}^{\prime} \max \left(C_{6}, C_{7}\right)\right\}\left(1-\kappa^{-1}\right)^{-1}$. We use Lemma 2.3 and Remark 2.4. So, if $\delta<2 \varepsilon$ and $1<\kappa \leq(2 \varepsilon) / \delta+\delta /(2 \varepsilon)-1$ we get

$$
\begin{aligned}
& \frac{v}{2 \kappa}\left(\frac{\beta \beta^{*} \delta \varepsilon}{\alpha \delta+2 \beta \varepsilon}\right)^{2} \int_{\Omega_{R}} u_{R}^{2} \eta_{\beta-1}^{\alpha-1} d x \leq \frac{v}{2 \kappa} \int_{\Omega_{R}}\left|\nabla u_{R}\right|^{2} \eta_{\beta}^{\alpha} d x \\
& \frac{v}{2 \kappa} \int_{\Omega_{R}}\left|\nabla u_{R}\right|^{2} \eta_{\beta}^{\alpha} d x+\frac{v}{2 \kappa}\left(\frac{\beta \beta^{*} \delta \varepsilon}{\alpha \delta+2 \beta \varepsilon}\right)^{2} \int_{\Omega_{R}} u_{R}^{2} \eta_{\beta-1}^{\alpha-1} d x \\
& \quad+k \delta \varepsilon(\beta-\alpha) \int_{\Omega_{R}} u_{R}^{2} \eta_{\beta-1}^{\alpha-1} s d x \leq C_{8} \int_{\Omega_{R}}|f|\left|u_{R}\right| \eta_{\beta}^{\alpha} d x
\end{aligned}
$$

So we get 


$$
\begin{aligned}
\int_{\Omega_{R}}\left|\nabla u_{R}\right|^{2} \eta_{\beta}^{\alpha} d x+2 \int_{\Omega_{R}} u_{R}^{2} \eta_{\beta-1}^{\alpha-1} d x+2 \varepsilon \int_{\Omega_{R}} u_{R}^{2} \eta_{\beta-1}^{\alpha-1} s d x \\
\quad \equiv \int_{\Omega_{R}}\left|\nabla u_{R}\right|^{2} \eta_{\beta}^{\alpha} d x+2 \int_{\Omega_{R}} u_{R}^{2} \eta_{\beta}^{\alpha-1} d x \leq C_{10} \int_{\Omega_{R}}|f|\left|u_{R}\right| \eta_{\beta}^{\alpha} d x
\end{aligned}
$$

where $\quad C_{10}=C_{8} / C_{9} \quad$ and $\quad C_{9}=\min \left(v /(2 \kappa),(v / 2 \kappa)\left(\left(\beta \beta^{*} \delta \varepsilon\right) /(\alpha \delta+2 \beta \varepsilon)\right)^{2}\right.$, $k \delta(\beta-\alpha) / 2)$. We have:

$$
C_{10} \int_{\Omega_{R}}|f|\left|u_{R}\right| \eta_{\beta}^{\alpha} d x \leq C_{10}\left(\frac{t}{2} \int_{\Omega} u_{R}^{2} \eta_{\beta}^{\alpha-1} d x+\frac{1}{2 t} \int_{\Omega} f^{2} \eta_{\beta}^{\alpha+1} d x\right) .
$$

So, if we choose $t=2 C_{10}^{-1}$ then we get

$$
\int_{\Omega_{R}}\left|\nabla u_{R}\right|^{2} \eta_{\beta}^{\alpha} d x+\int_{\Omega_{R}} u_{R}^{2} \eta_{\beta}^{\alpha-1} d x \leq C \int_{\Omega_{R}} f^{2} \eta_{\beta}^{\alpha+1} d x,
$$

where $C=C_{10}^{2} / 4$. It can be easily shown that the all conditions on $\alpha, \beta, \delta, \varepsilon, \kappa$ used in the proof are compatible if $0 \leq \alpha<y_{1} \beta$, see Appendix B.

\subsection{The model problem in $\Omega$}

Let $y_{1}$ be the same as in Lemma 3.4.

Theorem 3.5 (Existence and uniqueness). Let $0<\beta \leq 1,0 \leq \alpha<y_{1} \beta$, $f \in L_{\alpha+1, \beta}^{2}(\Omega)$ and $a \in\left(C^{(1)}(\bar{\Omega})\right)^{3}$. Let $\gamma=\gamma(\rho)$ be a nonnegative non-increasing continuous function defined on interval $[m,+\infty)$ with the limit $\lim _{\rho \rightarrow+\infty} \gamma(\rho)=0$, and the function a satisfies in $\Omega$ the following two conditions:

$$
\begin{aligned}
\operatorname{div}\left(\eta_{\beta_{0}}^{0} a\right) \leq & A \frac{v_{0}\left(\beta_{0}^{*}\right)^{2} \varepsilon_{0}^{2}}{4} \eta_{\beta_{0}-1}^{-1}+(1-A) \frac{v_{0}}{2^{3-\beta_{0}}} \frac{\eta_{\beta_{0}}^{0}}{r^{2}} \\
& +v(N-1) \frac{\left|\nabla \eta_{\beta_{0}}^{0}\right|^{2}}{\eta_{\beta_{0}}^{0}}
\end{aligned}
$$

for some $N>1, \quad 0 \leq A \leq 1, \quad 0<v_{0}<v, \quad 0<\beta_{0} \leq 1, \quad \varepsilon_{0}<(1 / 2) \cdot(k /(v N))$. $\left(1 / \beta_{0}\right), \eta_{\beta}^{\alpha} \equiv \eta_{\beta, \varepsilon_{0}}^{\alpha, \varepsilon_{0}}$.

$$
\operatorname{div}\left(\eta_{\beta}^{\alpha}(x) a(x)\right) \leq \gamma(|x|) \eta_{\beta-1}^{\alpha-1}(x),
$$

for $\eta_{\beta}^{\alpha} \equiv \eta_{\beta, \varepsilon}^{\alpha, \delta}$ with some $\delta, \varepsilon>0$. Then there exists a weak solution $u \in V_{\alpha, \beta}(\Omega)$ of the problem

$$
\begin{gathered}
-v \Delta u+k \partial_{1} u+a \cdot \nabla u=f \quad \text { in } \Omega, \\
u=0 \quad \text { on } \partial \Omega
\end{gathered}
$$


such that

$$
\|u\|_{2, \alpha-1, \beta}^{2}+\|\nabla u\|_{2, \alpha, \beta}^{2} \leq C\|f\|_{2, \alpha+1, \beta}^{2} .
$$

If moreover condition (3.36) is satisfied with $0<\beta_{0} \leq \beta$ and $-a \cdot \nabla r \leq$ const, $($ const $\in \boldsymbol{R})$ then there is a unique weak solution of problem (3.38), (3.39).

Proof. Existence. Let $R_{n} \subset \boldsymbol{R}, R_{n}>M, n \in \boldsymbol{N}$ be a sequence converging to $+\infty$. Let $u_{R_{n}}$ be the weak solution of (3.18), (3.19) on $\Omega_{R_{n}}$. Extending $u_{R_{n}}$ by zero on $\Omega-\Omega_{R_{n}}$ to a function $\tilde{u}_{n} \in V_{\alpha, \beta}(\Omega)$ we get a bounded sequence $\left\{\tilde{u}_{n}\right\}$ in $V_{\alpha, \beta}(\Omega)$. Thus, there is a subsequence $\tilde{u}_{n_{k}}$ of $\tilde{u}_{n}$ with a weak limit $u$ in $V_{\alpha, \beta}(\Omega)$. Obviously, $u$ is a weak solution of (3.38), (3.39) and

$$
\begin{aligned}
\|u\|_{2, \alpha-1, \beta}^{2}+\|\nabla u\|_{2, \alpha, \beta}^{2} & \leq \liminf _{k \in N}\left(\int_{\Omega} \tilde{u}_{n_{k}}^{2} \eta_{\beta}^{\alpha-1} d x+\int_{\Omega}\left|\nabla \tilde{u}_{n_{k}}\right|^{2} \eta_{\beta}^{\alpha} d x\right) \\
& \leq C \int_{\Omega}|f|^{2} \eta_{\beta}^{\alpha+1} d x .
\end{aligned}
$$

Uniqueness. In order to show uniqueness in $V_{\alpha, \beta}(\Omega)$ we will prove that the solution is unique in $V_{0, \beta_{0}}(\Omega) \supset V_{\alpha, \beta}(\Omega)$. Let $u \in V_{\alpha, \beta}(\Omega) \hookrightarrow V_{0, \beta_{0}}(\Omega)$ be a weak solution of (3.38), (3.39) with $f \equiv 0$. Let $\Phi=\Phi(z) \in C_{0}^{\infty}([0,+\infty))$ be a non-increasing cut-off function such that $\Phi(z) \equiv 1$ for $z<1 / 2$ and $\Phi(z) \equiv 0$ for $z>1$. Let $\left|\Phi^{\prime}\right| \leq 3$. Let $\Phi_{R} \equiv \Phi_{R}(x) \equiv \Phi(r / R) \equiv \Phi(|x| / R)$ for $x \in \Omega$. We have $\left|\nabla \Phi_{R}\right| \leq 3 / R$ and $\left|\partial_{1} \Phi_{R}\right| \leq 3 / R$ on $\Omega_{R}^{R / 2}$. Let $\left\{R_{j}\right\} \in R$ be an increasing sequence of radii with the limit $+\infty$. So we have that $u_{j} \equiv u \Phi_{R_{j}} \in \stackrel{\circ}{H}^{1}(\Omega)$. So, $\left\{u_{j}\right\}$ is a sequence of functions with limit $u$ in the space $V_{\alpha, \beta}(\Omega)$. Using the test functions $\varphi=u \Phi_{R_{j}}^{2} \eta_{\beta_{0}}^{0}=u_{j} \Phi_{R_{j}}\left(1+\varepsilon_{0} s\right)^{\beta_{0}} \in \stackrel{\circ}{H^{1}}(\Omega)$ in (3.29) we have:

$$
\begin{gathered}
v \int_{\Omega} \nabla u \cdot \nabla\left(u \Phi_{R_{j}}^{2} \eta_{\beta_{0}}^{0}\right) d x+k \int_{\Omega} \partial_{1} u u \Phi_{R_{j}}^{2} \eta_{\beta_{0}}^{0} d x \\
+\int_{\Omega} a \cdot \nabla u u \Phi_{R_{j}}^{2} \eta_{\beta_{0}}^{0} d x=0 .
\end{gathered}
$$

Using in (3.40) relations

$$
\begin{gathered}
\nabla u \cdot \nabla\left(u \Phi_{R_{j}}^{2} \eta_{\beta_{0}}^{0}\right)=\left|\nabla u_{j}\right|^{2} \eta_{\beta_{0}}^{0}-\nabla \Phi_{R_{j}} \cdot \nabla \Phi_{R_{j}} u^{2} \eta_{\beta_{0}}^{0}+\nabla u \cdot \nabla \eta_{\beta_{0}}^{0} \Phi_{R_{j}}^{2} u \\
u \nabla u \cdot \nabla \eta_{\beta_{0}}^{0} \Phi_{R_{j}}^{2}=u_{j} \nabla u_{j} \cdot \nabla \eta_{\beta_{0}}^{0}-u^{2} \nabla \Phi_{R_{j}} \cdot \nabla \eta_{\beta_{0}}^{0} \Phi_{R_{j}}
\end{gathered}
$$

integrating by parts, we get after some evident rearrangements and (3.36):

$$
\begin{aligned}
E\left(u_{j}, \Omega_{R_{j}}\right) \leq & \frac{k}{2} \int_{\Omega} u^{2} \partial_{1} \Phi_{R_{j}}^{2} \eta_{\beta_{0}}^{0} d x+\frac{1}{2} \int_{\Omega} u^{2} \eta_{\beta_{0}}^{0} a \cdot \nabla \Phi_{R_{j}}^{2} d x \\
& +v \int_{\Omega}\left|\nabla \Phi_{R_{j}}\right|^{2} u^{2} \eta_{\beta_{0}}^{0} d x+v \int_{\Omega} u^{2} \nabla \Phi_{R_{j}} \cdot \nabla \eta_{\beta_{0}}^{0} \Phi_{R_{j}} d x
\end{aligned}
$$


where $E\left(v, \Omega_{R}\right)$ was defined by (3.24). Let us show the estimate of the integral $(1 / 2) \int_{\Omega} u^{2} \eta_{\beta_{0}}^{0} a \cdot \nabla \Phi_{R_{j}}^{2} d x$, (the other integrals on the right hand side can be estimated similarly but more simply by the integral $\int_{\Omega_{R_{j}}^{R_{j} / 2}} u^{2} \eta_{\beta_{0}}^{-1} d x$ ):

$$
\begin{aligned}
& \frac{1}{2} \int_{\Omega} u^{2} \eta_{\beta_{0}}^{0} a \cdot \nabla \Phi_{R_{j}}^{2} d x=\frac{1}{2} \int_{\Omega}\left(u^{2} \eta_{\beta_{0}}^{0} a\right) \cdot\left[2 \Phi_{R_{j}} \Phi^{\prime}\left(\frac{r}{R_{j}}\right) \frac{1}{R_{j}} \nabla r\right] d x \\
& \quad \leq 3 \int_{\Omega_{R_{j}}^{R_{j} / 2}}\left(u^{2} \eta_{\beta_{0}}^{0} \frac{1}{R_{j}}\right)(-1) \min (0, a \cdot \nabla r) d x \\
& \quad \leq 3 c \int_{\Omega_{R_{j}}^{R_{j} / 2}}\left(u^{2} \eta_{\beta_{0}}^{-1}\right) \max (0,-a \cdot \nabla r) d x \equiv 3 c \int_{\Omega_{R_{j}}^{R_{j} / 2}} u^{2} \eta_{\beta_{0}}^{-1}[a \cdot \nabla r]_{-} d x
\end{aligned}
$$

where $[y]_{-}$means the negative part of a real number $y$, i.e. $[y]_{-} \equiv \max (0,-y)$. (In the first inequality we used $\Phi^{\prime} \leq 0,\left|\Phi^{\prime}\right| \leq 3, \Phi_{R_{j}} \geq 0$ on $\Omega, \Phi^{\prime} \equiv 0$ on $\Omega \backslash \Omega_{R_{j}}^{R_{j} / 2}$, the second inequality follows from $1 / R_{j} \leq c /(1+\delta r)$ on $\Omega_{R_{j}}^{R_{j} / 2}$ for $c>\delta$ if $R_{j} \geq 1 /(c-\delta)$.) So, estimating $E\left(u_{j}, \Omega_{R_{j}}\right)$ from below as in the proof of Lemma 3.2, we get:

$$
\begin{gathered}
\left(1-\frac{1}{\kappa}\right) \frac{v}{2} \int_{\Omega}\left|\nabla u_{j}\right|^{2} \eta_{\beta_{0}}^{0} d x+\frac{1}{2}\left(1-\frac{1}{\kappa}\right) k \varepsilon_{0}^{2} \beta_{0} \int_{\Omega} u_{j}^{2} \eta_{\beta_{0}-1}^{-1} s d x \\
\leq C\left(\int_{\Omega_{R_{j}}^{R_{j} / 2}} u^{2} \eta_{\beta_{0}}^{-1} d x+\int_{\Omega_{R_{j}}^{R_{j} / 2}} u^{2} \eta_{\beta_{0}}^{-1}[a \cdot \nabla r]_{-} d x\right)
\end{gathered}
$$

For $j \rightarrow \infty$ we get $(-a \cdot \nabla r \leq$ const $)$ :

$$
v \int_{\Omega}|\nabla u|^{2} \eta_{\beta_{0}}^{0} d x+k \varepsilon_{0}^{2} \beta_{0} \int_{\Omega} u^{2} \eta_{\beta_{0}-1}^{-1} s d x \leq 0
$$

So, the uniqueness was proved.

Remark 3.6. The right hand side $f$ in (3.38) in Theorem 3.5 can be chosen from the dual space of $V_{\alpha, \beta}(\Omega)$ : Let $f \in H_{l o c}^{-1}(\bar{\Omega})$ be a functional such that $\|f\|_{-1, \alpha, \beta}<+\infty$, where

$$
\|v\|_{-1, \alpha, \beta} \equiv \sup \left\{\left|\left\langle f, \eta_{\beta}^{\alpha} v\right\rangle\right| ; v \in V_{\alpha, \beta}(\Omega),\|v\|_{2, \alpha-1, \beta}+\|\nabla v\|_{2, \alpha, \beta}=1\right\} .
$$

One can get in this case the estimate $\|u\|_{2, \alpha-1, \beta}^{2}+\|\nabla u\|_{2, \alpha, \beta}^{2} \leq C^{\prime}\|f\|_{-1, \alpha, \beta}^{2}$.

\subsection{Sufficient conditions on $a(\cdot)$}

Conditions (3.36), (3.37) are given in the form which is appropriate for the proof. Now, we will formulate simpler sufficient conditions ensuring these relations. We shall denote in the next lemma $\eta_{\beta}^{\alpha} \equiv \eta_{\beta, 1}^{\alpha, 1}$. 
Lemma 3.7. Let $a \in C^{(1)}(\bar{\Omega})$ satisfies at least one of the conditions A)-C):

A) There are $K>0, d>0$ and $0<v_{0}<v$ such that in $\Omega$

$$
a \cdot \frac{\nabla s}{|\nabla s|} \leq K \eta_{-1 / 4+d}^{-3 / 4-d}, \quad \operatorname{div} a \leq \frac{v_{0}}{2^{4-\beta_{0}}} \frac{1}{r^{2}} .
$$

B) There are $K>0,0<d<1 / 2$ and $1<\kappa<\left[1+(v /(k K))^{1 / d}\right]^{2 d}$ such that in $\Omega$

$$
a \cdot \frac{\nabla s}{|\nabla s|} \leq K \eta_{-1 / 4+d}^{-3 / 4-d}, \quad \operatorname{div} a \leq \frac{k}{8} \frac{\kappa-1}{\kappa^{2}} \eta_{-1}^{-1} .
$$

C) There are $A \geq 0, B \geq 0, A+B<1$ such that in $\Omega$

$$
a \cdot \frac{\nabla s}{|\nabla s|} \leq A \min \left(\beta_{0} v, \frac{k}{4}\right) \eta_{-1 / 2}^{-1 / 2}, \quad \operatorname{div} a \leq \frac{B v}{16} \min \left(4 \beta_{0}^{2}, \frac{k^{2}}{4 v^{2}}\right) \eta_{-1}^{-1} .
$$

Then condition (3.21) is satisfied.

Proof. A) Let in this proof $\eta_{\beta}^{\alpha} \equiv \eta_{\beta, \varepsilon_{0}}^{\alpha, \varepsilon_{0}}$. Expressing explicitly terms of the relation (3.36) we get

$$
\begin{gathered}
\eta_{\beta_{0}}^{0} \operatorname{div} a+(a \cdot \nabla s) \beta_{0} \varepsilon_{0} \eta_{\beta_{0}-1}^{0} \leq A \frac{v_{0}\left(\beta_{0}^{*}\right)^{2} \varepsilon_{0}^{2}}{4} \eta_{\beta_{0}-1}^{-1} \\
+(1-A) \frac{v_{0}}{2^{3-\beta_{0}}} \frac{\eta_{\beta_{0}}^{0}}{r^{2}}+v(N-1) \beta_{0}^{2} \varepsilon_{0}^{2} \frac{2 s}{r} \eta_{\beta_{0}-2}^{0}
\end{gathered}
$$

It is clear that the following conditions (3.41) and (3.42) imply (3.36):

$$
\begin{aligned}
a \cdot \nabla s \leq & B\left(A \frac{v_{0} \beta_{0} \varepsilon_{0}}{4} \eta_{0}^{-1}+(1-A) \frac{v_{0}}{4 \cdot 2^{1-\beta_{0}} \beta_{0} \varepsilon_{0} r^{2}} \eta_{1}^{0}\right) \\
& +v(N-1) \beta_{0} \varepsilon_{0} \frac{2 s}{r} \eta_{-1}^{0} \\
\operatorname{div} a \leq & (1-B)\left(A \frac{v_{0}\left(\beta_{0}^{*}\right)^{2} \varepsilon_{0}^{2}}{4} \eta_{-1}^{-1}+(1-A) \frac{v_{0}}{4 \cdot 2^{1-\beta_{0}} r^{2}}\right)
\end{aligned}
$$

with $0 \leq A \leq 1,0 \leq B \leq 1$. Taking $A=1 / 2-2 d$ with $0<d<1 / 4$ we estimate the right hand side in (3.41) using the inequality $F^{t} G^{1-t} \leq t F+(1-t) G$ for $F, G \geq 0,0 \leq t \leq 1$ :

$$
\begin{aligned}
B(A & \left.\frac{v_{0} \beta_{0} \varepsilon_{0}}{4} \eta_{0}^{-1}+(1-A) \frac{v_{0}}{2^{3-\beta_{0}} \beta_{0} \varepsilon_{0} r^{2}} \eta_{1}^{0}\right)+v(N-1) \beta_{0} \varepsilon_{0} \frac{2 s}{r} \eta_{-1}^{0} \\
\geq & {\left[B(N-1) v_{0} v\right]^{1 / 2}\left(\frac{1}{2^{1-\beta_{0}}}\right)^{1 / 4+d}\left(\beta_{0} \varepsilon_{0}\right)^{1 / 2-2 d}\left(\frac{2 s}{r}\right)^{1 / 2} r^{-1 / 2-2 d} \eta_{-1 / 4+d}^{-1 / 4+d} } \\
\geq & {\left[B(N-1) v_{0} v\right]^{1 / 2}\left(\frac{1}{2^{1-\beta_{0}}}\right)^{1 / 4+d}\left(\beta_{0} \varepsilon_{0}\right)^{1 / 2-2 d} } \\
& \cdot|\nabla s| \min \left(1, \varepsilon_{0}^{-1 / 2+2 d}\right) \eta_{-1 / 4+d, 1}^{-3 / 4-d, 1}
\end{aligned}
$$


Let us take some $\kappa>1$ and $N \equiv N\left(\varepsilon_{0}\right)=(1 / 2) \cdot\left(k /\left(\kappa v \varepsilon_{0}\right)\right) \cdot\left(1 / \beta_{0}\right)$, so the condition $\varepsilon_{0}<(1 / 2) \cdot(k /(v N)) \cdot\left(1 / \beta_{0}\right)$ is satisfied. Evidently, for $\varepsilon_{0} \rightarrow 0$ we have $(N-1)^{1 / 2}\left(\beta_{0} \varepsilon_{0}\right)^{1 / 2-2 d} \rightarrow+\infty$. Hence for arbitrary $B>0, K>0,0<\beta_{0} \leq 1$ there is $\varepsilon_{0}>0$ and $N \equiv N\left(\varepsilon_{0}\right)>1$ such that the expression (3.43) can be estimated from below:

$$
\geq K|\nabla s| \eta_{-1 / 4+d, 1}^{-3 / 4-d, 1}
$$

Hence, the sufficient condition for (3.41) is

$$
a \cdot \frac{\nabla s}{|\nabla s|} \leq K \eta_{-1 / 4+d, 1}^{-3 / 4-d, 1}
$$

We estimate now the right hand side in (3.42). Because, $B>0$ can be taken sufficiently small and $A=1 / 2-2 d$, we have:

$$
\begin{gathered}
(1-B)\left(A \frac{v_{0}\left(\beta_{0}^{*}\right)^{2} \varepsilon_{0}^{2}}{4} \eta_{-1, \varepsilon_{0}}^{-1, \varepsilon_{0}}+(1-A) \frac{v_{0}}{2^{3-\beta_{0} r^{2}}}\right) \\
\geq(1-B)\left(\frac{1}{2}+2 d\right) \frac{v_{0}}{2^{3-\beta_{0} r^{2}}} \geq \frac{v_{0}}{2^{4-\beta_{0} r^{2}}}
\end{gathered}
$$

So, sufficient condition for $(3.42)$ is

$$
\operatorname{div} a \leq \frac{v_{0}}{2^{4-\beta_{0} r^{2}}}
$$

Analogously we can get conditions B) and C).

In the next lemma we will present the sufficient condition ensuring condition (3.30) of Lemma 3.4

Lemma 3.8. Let $a \in\left(C^{(1)}(\bar{\Omega})\right)^{3}$ and let $\gamma_{0}=\gamma_{0}(\rho)$ be a nonnegative nonincreasing continuous function defined on interval $[m,+\infty)$ with the limit $\lim _{\rho \rightarrow+\infty} \gamma(\rho)=0$, and the function a satisfies the following conditions:

$$
\operatorname{div} a \leq \gamma_{0}(r) \eta_{-1}^{-1}, \quad a \cdot \frac{\nabla s}{|\nabla s|} \leq \gamma_{0}(r) \eta_{-1 / 2}^{-1 / 2}, \quad a \cdot \nabla r \equiv a \cdot \frac{\nabla r}{|\nabla r|} \leq \gamma_{0}(r) \eta_{-1}^{0}
$$

Then condition (3.30) is satisfied.

Proof. We proceed analogously as in the proof of Lemma 3.7.

From Theorem 3.5, Lemma 3.7 and Lemma 3.8 follows 
Theorem 3.9. Let $0<\beta \leq 1,0 \leq \alpha<y_{1} \beta, f \in L_{\alpha+1, \beta}^{2}(\Omega)$ and $a \in\left(C^{(1)}(\bar{\Omega})\right)^{3}$. Let $\gamma_{0}=\gamma_{0}(\rho)$ be a nonnegative non-increasing continuous function defined on interval $[m,+\infty)$ with the limit $\lim _{\rho \rightarrow+\infty} \gamma_{0}(\rho)=0$. We assume one of the following cases, $\left(\eta_{\beta}^{\alpha} \equiv \eta_{\beta, 1}^{\alpha, 1}\right.$, const $\left.\in \boldsymbol{R}\right)$ :

i) There are $K>0, d>0$ and $0<v_{0}<v$ such that in $\Omega$ :

$a \cdot \frac{\nabla s}{|\nabla s|} \leq K \eta_{-1 / 4+d}^{-3 / 4-d}, \quad \operatorname{div} a \leq \frac{v_{0}}{2^{4-\beta}} \frac{1}{r^{2}}, \quad$ const $\leq a \cdot \nabla r \leq \gamma_{0}(|x|) \eta_{-1}^{0}$.

ii) There are $K>0,0<d<1 / 2$ and $1<\kappa<\left[1+(v /(k K))^{1 / d}\right]^{2 d}$ such that in $\Omega$ :

$$
\begin{gathered}
a \cdot \frac{\nabla s}{|\nabla s|} \leq K \eta_{-1 / 4+d}^{-3 / 4-d}, \quad \text { const } \leq a \cdot \nabla r \leq \gamma_{0}(r) \eta_{-1}^{0} \\
\operatorname{div} a \leq \frac{1}{8} \frac{\kappa-1}{\kappa^{2}} k \eta_{-1}^{-1}, \quad \operatorname{div} a \leq \gamma_{0}(r) \eta_{-1}^{-1}
\end{gathered}
$$

iii) There are $A \geq 0, B \geq 0, A+B<1$ such that in $\Omega$ :

$$
\begin{gathered}
a \cdot \frac{\nabla s}{|\nabla s|} \leq A \min \left(\beta v, \frac{k}{4}\right) \eta_{-1 / 2}^{-1 / 2}, \quad \operatorname{div} a \leq \frac{B v}{16} \min \left(4 \beta^{2}, \frac{k^{2}}{4 v^{2}}\right) \eta_{-1}^{-1}, \\
\operatorname{div} a \leq \gamma_{0}(r) \eta_{-1}^{-1}, \quad a \cdot \frac{\nabla s}{|\nabla s|} \leq \gamma_{0}(r) \eta_{-1 / 2}^{-1 / 2}, \quad \text { const } \leq a \cdot \nabla r \leq \gamma_{0}(r) \eta_{-1}^{0} .
\end{gathered}
$$

Then there exists a unique weak solution $u \in V_{\alpha, \beta}(\Omega)$ of the problem (1.1), (1.2) such that

$$
\|u\|_{2, \alpha-1, \beta}^{2}+\|\nabla u\|_{2, \alpha, \beta}^{2} \leq C\|f\|_{2, \alpha+1, \beta}^{2} .
$$

\section{Appendix A}

Let us recall the definition of function $F_{\alpha, \beta}(s, r ; v), r>0, s \in[0,2 r]$ :

$$
F_{\alpha, \beta}(s, r ; v) \equiv\left(-v \frac{\left|\nabla \eta_{\beta}^{\alpha}\right|^{2}}{\eta_{\beta}^{\alpha}}-k \partial_{1} \eta_{\beta}^{\alpha}\right) / \eta_{\beta-1}^{\alpha-1}
$$

Lemma 3.10. Let $0 \leq \alpha<\beta$. Then, (for a sufficiently small $\delta>0$ ) there exists $s_{0}>0$ such that

$$
F_{\alpha, \beta}(s, r ; v) \geq 0
$$

for all $r>0, \quad s \geq s_{0}>0$. Let moreover $\quad \kappa>1, \quad 0<\varepsilon \leq(1 /(2 \kappa)) \cdot(k / v)$. $\left((\beta-\alpha) / \beta^{2}\right)$ and $\delta, v, k>0$. Then 


$$
F_{\alpha, \beta}(s, r ; v)-\left(1-\frac{1}{\kappa}\right) k \delta \varepsilon(\beta-\alpha) s \geq-\alpha \delta k\left(1+\frac{v}{k} \alpha \delta\right)
$$

for all $r>0$ and $s \in\left[0, s_{0}\right)$.

Proof. First, let us assume $\alpha>0$. We have:

$$
\begin{aligned}
F_{\alpha, \beta}(s, r ; v)= & -v \alpha^{2} \delta^{2}\left(\frac{1+\varepsilon s}{1+\delta r}\right)-2 v \alpha \beta \delta \varepsilon \frac{s}{r}-2 v \beta^{2} \varepsilon^{2}\left(\frac{1+\delta r}{1+\varepsilon s}\right) \frac{s}{r} \\
& -k \alpha \delta(1+\varepsilon s) \frac{x_{1}}{r}+k \beta \varepsilon(1+\delta r) \frac{s}{r}
\end{aligned}
$$

where $x_{1}=r-s$. So, the expression of $r(1+\delta r)(1+\varepsilon s) F_{\alpha, \beta}(s, r ; v)$ is the polynomial of $R=1+\delta r$ with coefficients being polynomials of $S=1+\varepsilon s$ :

$$
r(1+\delta r)(1+\varepsilon s) F_{\alpha, \beta}(s, r ; v)=A R^{2}+B R+C,
$$

where $\quad A=k(\beta-\alpha) S^{2}+\left(-2 v \beta^{2} \varepsilon-k \beta\right) S+2 v \beta^{2} \varepsilon$,

$$
\begin{aligned}
& B=k \alpha \frac{\delta}{\varepsilon} S^{3}+\left(k \alpha\left(1-\frac{\delta}{\varepsilon}\right)-v \alpha \delta(2 \beta+\alpha)\right) S^{2}+2 v \alpha \beta \delta S, \\
& C=v \alpha^{2} \delta S^{2}
\end{aligned}
$$

Note that the discriminant $D$ of polynomial $A$ is positive for all $\alpha, \beta, \delta, \varepsilon, v$, $k>0$. We can see that $C>0, B>0$ for $\delta$ small enough (if $k \alpha>v \alpha \delta(2 \beta+\alpha)$ ), and $A>0$ if $S>S_{0}$, where $S_{0}=(1 /(2 k(\beta-\alpha)))\left[2 v \beta^{2} \varepsilon+k \beta+\sqrt{D}\right]$. Finally, let us denote $s_{0}=\left(S_{0}-1\right) / \varepsilon$, then

$$
F_{\alpha, \beta}(s, r ; v) \geq 0
$$

for all $r>0$, if $s \geq s_{0}$. Let us mention that if $\varepsilon$ is small enough, i.e. if $0<\varepsilon<k /(2 v \beta)$, we have $s_{0} \leq(\alpha /(\varepsilon(\beta-\alpha)))\left(1+2 v k \beta \varepsilon /(k-2 v \beta \varepsilon)^{2}\right)$. Indeed,

$$
\begin{aligned}
S_{0}= & \frac{1}{2 k(\beta-\alpha)}\left[\left(2 v \beta^{2} \varepsilon+k \beta\right)+\sqrt{D}\right]=\frac{\beta}{(\beta-\alpha)}\left[\left(\frac{v \beta \varepsilon}{k}+\frac{1}{2}\right)\right. \\
& \left.+\left(\frac{1}{2}-\frac{v \beta \varepsilon}{k}\right) \sqrt{1+\frac{8 v k \varepsilon \alpha}{(k-2 v \beta \varepsilon)^{2}}}\right] \leq \frac{\beta}{(\beta-\alpha)}\left[1+\frac{2 v k \varepsilon \alpha}{(k-2 v \beta \varepsilon)^{2}}\right], \\
s_{0}= & \frac{1}{\varepsilon}\left(S_{0}-1\right) \leq \frac{1}{\varepsilon} \frac{\alpha}{(\beta-\alpha)}\left(1+\frac{2 v k \beta \varepsilon}{(k-2 v \beta \varepsilon)^{2}}\right) .
\end{aligned}
$$

Relation (3.45) follows from an estimate of the derivative of function $F_{1}$ : 


$$
\begin{aligned}
F_{1}(s, r) \equiv & F_{\alpha, \beta}(s, r ; v)-\left(1-\frac{1}{\kappa}\right) k \delta \varepsilon(\beta-\alpha) s \\
\frac{\partial}{\partial s} F_{1}(s, r)= & -v \alpha^{2} \delta^{2} \varepsilon \frac{1}{1+\delta r}-2 v \alpha \beta \delta \varepsilon \frac{1}{r}-2 v \beta^{2} \varepsilon^{2} \frac{1+\delta r}{r} \frac{1}{(1+\varepsilon s)^{2}} \\
& -k \alpha \delta \varepsilon+k \alpha \delta \frac{1}{r}(1+2 \varepsilon s)+k \beta \varepsilon(1+\delta r) \frac{1}{r} \\
& -\left(1-\frac{1}{\kappa}\right) k \delta \varepsilon(\beta-\alpha), \\
\frac{\partial}{\partial s} F_{1}(s, r) \geq & \delta \varepsilon\left\{\frac{1}{r}\left[k\left(\frac{\alpha}{\varepsilon}+\frac{\beta}{\delta}\right)-v \alpha^{2}-2 v \alpha \beta-2 \frac{v \beta^{2} \varepsilon}{\delta}\right]\right. \\
& \left.+\left[-2 v \beta^{2} \varepsilon+\frac{1}{\kappa} k(\beta-\alpha)\right]\right\} .
\end{aligned}
$$

We have $k \alpha / \varepsilon \geq v \alpha^{2}+2 v \alpha \beta, \quad k \beta / \delta \geq 2 v \beta^{2} \varepsilon / \delta, \quad k(\beta-\alpha) / \kappa \geq 2 v \beta^{2} \varepsilon \quad$ if $\varepsilon \leq$ $k(\beta-\alpha) /\left(2 \kappa v \beta^{2}\right)$. Hence, if $\varepsilon \leq k(\beta-\alpha) /\left(2 \kappa v \beta^{2}\right)$ then

$$
\frac{\partial}{\partial s} F_{1}(s, r) \geq 0
$$

So, we get immediately:

$$
F_{1}(s, r) \geq F_{1}(0, r) \equiv-k \alpha \delta-v \alpha^{2} \delta^{2} \frac{1}{1+\delta r} \geq-\alpha \delta k\left(1+\frac{v}{k} \alpha \delta\right) .
$$

\section{Appendix B}

Let us show that all conditions on $\alpha, \beta, \delta, \varepsilon, \kappa$ used in the proof of Lemma 3.4 are compatible if $0<\beta \leq 1,0 \leq \alpha<y_{1} \beta$. Let us collect these assumptions: $0<\delta<2 \varepsilon, 1<\kappa \leq(2 \varepsilon) / \delta+\delta /(2 \varepsilon)-1,0 \leq \alpha<\beta, \varepsilon \leq\left(1 /\left(2 \kappa^{2}\right)\right) \cdot(k / v)$. $\left((\beta-\alpha) / \beta^{2}\right), \delta \leq(k / v) \cdot(\kappa-1) /(\kappa \beta), \alpha \leq\left(1 /\left(2 \kappa^{4}\right)\right) \cdot(v / k) \cdot\left(\beta \beta^{*} /(\alpha+\beta)\right)^{2} \varepsilon$.

From $\alpha \leq\left(1 /\left(2 \kappa^{4}\right)\right) \cdot(v / k) \cdot\left(\beta \beta^{*} /(\alpha+\beta)\right)^{2} \varepsilon$, and $\varepsilon \leq\left(1 /\left(2 \kappa^{2}\right)\right) \cdot(k / v)$. $\left((\beta-\alpha) / \beta^{2}\right)$ we get $\alpha \leq\left(1 /\left(4 \kappa^{6}\right)\right) \cdot\left(\beta^{*}\right)^{2}(\beta-\alpha) /(\alpha+\beta)^{2}$. So we get $(\kappa>1$, $\beta \leq 1): \quad \alpha / \beta \leq\left(1 /\left(4 \kappa^{6}\right)\right)(1-\alpha / \beta) /(1+\alpha / \beta)^{2}$. By substitution $y=\alpha / \beta$ we get the inequality

$$
4 y^{3}+8 y^{2}+4 y+\kappa^{-6}(y-1) \leq 0 .
$$

Taking into account the condition $0 \leq \alpha<\beta$ we seek for solutions from $[0,1)$. It is clear that the equation $4 y^{3}+8 y^{2}+4 y+\kappa^{-6}(y-1)=0$ has a unique real solution $y_{\kappa} \in(0,1)$ for $\kappa>1$. It is also clear that arbitrary 
$y \in\left[0, y_{\kappa}\right)$ solves (3.46). The value $y_{\kappa}$ as a function of $\kappa$ is decreasing. For $\kappa \rightarrow 1$ we get the inequality $4 y^{3}+8 y^{2}+5 y-1 \leq 0$. This respective equation has a unique solution $y_{1}=(\sqrt{13} /(6 \sqrt{6})+53 / 216)^{1 / 3}+(1 / 30)(\sqrt{13} /(6 \sqrt{6})+$ $53 / 216)^{-1 / 3}$. Approximately, with an error less than $10^{-8}$ we have $y_{1} \doteq$ $0.1582981\left(y_{1}>1 / 7\right)$. If $0 \leq \alpha<y_{1} \beta$ then there is $\kappa>1$ sufficiently close to number 1 , such that $0 \leq \alpha \leq y_{\kappa} \beta$, so the relation $\alpha \leq\left(1 /\left(4 \kappa^{6}\right)\right) \cdot\left(\beta^{*}\right)^{2}(\beta-\alpha) /$ $(\alpha+\beta)^{2}$ is satisfied. Then we can define $\varepsilon=\left(1 /\left(2 \kappa^{2}\right)\right) \cdot(k / v) \cdot\left((\beta-\alpha) / \beta^{2}\right)$. The relation $\varepsilon \leq(1 / 2 \kappa) \cdot(k / v) \cdot(1 / \beta)$ is satisfied. Then we take sufficiently small $\delta>0$ such that $0<\delta<2 \varepsilon$ and $1<\kappa \leq(2 \varepsilon) / \delta+\delta /(2 \varepsilon)-1$. Hence, all conditions which we assume in the proof of Lemma 3.4 are satisfied.

\section{Acknowledgement}

The authors gratefully appreciate the hospitality of the Mathematical Institute of the Academy of Sciences of the Czech Republic and the University in Toulon. The first author is supported by grant No. 201/02/0684 from the Grant Agency of the Czech Republic, and partly supported by grant No. A2120201/02 from the Academy of Sciences of the Czech Republic.

\section{References}

[1] Adams R. A., Sobolev Spaces, Academic Press, New York, 1975.

[2] Farwig R., A variational approach in weighted Sobolev spaces to the operator $-\Delta+\partial / \partial_{1}$ in exterior domains of $\boldsymbol{R}^{3}$. Mathematische Zeitschrift 210 (1992), 449-464.

[3] Farwig R., The stationary exterior 3-D problem of Oseen and Navier-Stokes equations in anisotropically weighted Sobolev spaces, Mathematische Zeitschrift 211 (1992), 409-447.

[4] Finn R., Estimates at infinity for stationary solution of Navier-Stokes equations, Bult. Math. de la Soc. Sci. Math. de la R.P.R. Tome 3, 53 (4) (1959), 387-418.

[5] Finn R., On the Exterior Stationary Problem and Associated Perturbation Problems for the N.S. Eq's, Arch. Rat. Mech. Anal. 19 (1965), 363-406.

[6] Hishida, T., The Stokes operator with rotation effect in exterior domains, Analysis 19 (1999), 51-67.

[7] Impagliazzo, J.-L., Resolution des equations de Navier-Stokes compressibles a l'aide de la methode de decomposition, These de doctorat de l'Universite de Toulon et du Var, 1997.

[8] Kobayashi T., Shibata Y., On the Oseen equation in the three-dimensional exterior domains, Math. Ann. 310 (1998), 1-45.

[ 9 ] Kufner A., Weighted Sobolev Spaces, BSB B. G. Teubner Verlagsgesellschaft, Leipzig, 1980.

[10] Kračmar S., Novotný A., Pokorný M., Estimates of three dimensional Oseen kernels in weighted $L^{p}$ spaces, In: "Applied Nonlinear Analysis," 281-316. London-New York, Plenum Publishers/Kluwer Academic, 1999.

[11] Kračmar S., Novotný A., Pokorný M., Estimates of Oseen kernels in weighted $L^{p}$ spaces, J. Math. Soc. Japan 53, No. 1 (2001), 59-111.

[12] Nečasová, Š., Asymptotic properties of the steady fall of a body in a viscous fluid, Mathem. Models and Appl. (to appear). 
[13] Novotný A., Pokorný M., Three-dimensional steady flow of viscoelastic fluid past an obstacle, J. Math. Fluid Mech. 2 (2000), No. 3, 294-414.

[14] Novotný A., Pokorný M., Steady plane flow of viscoelastic fluid past an obstacle, Applications of Math. 47 (2002), 231-254.

[15] Oseen C. W., Neuere Methoden und Ergebnisse in der Hydrodynamik, Leipzig, Akad. Verlagsgesellschaft M.B.H., 1927.

[16] Rudin W., Real and Complex Analysis, McGraw-Hill, New York, second edition, 1974.

[17] Smith D., Estimates at Infinity for Stationary Solutions of the N.S. Equations in Two Dimensions, Arch. Rat. Mech. Anal. 20 (1965), 341-372.

[18] Turesson B. O., Nonlinear Potential Theory and Weighted Sobolev Spaces, Springer Verlag, New York, 2000.

nuna adreso:

S. Kračmar

Department of Technical Mathematics

Faculty of Mechanical Engineering

Czech Technical University

Karlovo nám. 13, 12135 Prague

Czech Republic

E-mail: kracmar@fsik.cvut.cz

P. Penel

Department of Mathematics

Université de Toulon et du Var

B.P. 132, 83957 Toulon-La Garde

France

E-mail: penel@univ-tln.fr

(Ricevita la 17-an de septembro, 2003)

(Reviziita la 2-an de februaro, 2004) 